\title{
Adiabatic following model for two-photon transitions: Nonlinear mixing and pulse propagation
}

\author{
D. Grischkowsky* and M. M. T. Loy \\ IBM Thomas J. Watson Research Center, Yorktown Heights, New York 10598
}

\author{
P. F. Liao \\ Bell Telephone Laboratories, Holmdel, New Jersey 07733
}

(Received 14 July 1975)

\begin{abstract}
Two-photon resonantly enhanced parametric generation processes have generally been described using timedependent perturbation theory. In this paper we show that a theory of two-photon coherent effects can be used to derive and explain these nonlinear mixing processes. Our technique makes use of the adiabatic following (AF) approximation to obtain solutions to a vector model describing the two-photon resonance. We show that the usual results for the nonlinear susceptibilities correspond to the $\vec{r}$ vector of Feynman, Vernon, and Hellwarth adiabatically following the $\vec{\gamma}$ vector in the small-angle limit. Consequently, the theory allows a natural extension to large angles, and power-dependent nonlinear susceptibilities are obtained. We then use these AF results for the polarization to study the propagation of pulses nearly resonant with a two-photon transition, and we demonstrate that the pulse reshaping is due to the two related effects of a nonlinear pulse velocity and self-phase modulation.
\end{abstract}

\section{INTRODUCTION}

The vector model of Feynman, Vernon, and Hellwarth ${ }^{1}$ has been a powerful tool in understanding many coherent phenomena involving the interaction of light with two-level systems. Recently it has been shown that two-photon processes can also be interpreted in terms of similar vector models. ${ }^{2,3}$ Here we show that, by applying the adiabatic following (AF) approximation ${ }^{4-8}$ to a two-photon vector model, ${ }^{2}$ explicit expressions for the power-dependent nonlinear susceptibilities are obtained. Also, the AF model is used to derive a nonlinear pulse velocity for pulses nearly resonant with a two-photon transition and to calculate the large self-phase modulation simultaneously obtained.

The nonlinear mixing processes were explained and the nonlinear coefficients derived in the classic paper by Armstrong et al. in 1962. ${ }^{9}$ These derivations were made with time-dependent second-order perturbation theory. Recently, twophoton resonant enhancement of the nonlinear coefficients has been shown to increase dramatically the efficiency of third-harmonic generation and other parametric generation processes. ${ }^{10^{-12}}$ However, the limitations of perturbation theory as the two-photon resonance is approached are not well understood.

The theory of two-photon coherent effects was separately developed. The first paper in this area was written in 1968 by Hartmann, ${ }^{13}$ where he developed the theory of the Raman echo for a threelevel system. Then in 1969, Belenov and Poluektov $^{14}$ studied theoretically the propagation of an optical pulse resonant with a two-photon transition. They recast the general multilevel problem with a two-level approximation and derived the essential results. Later in 1971, these results were reobtained by Takatsuji ${ }^{2}$ using density-matrix techniques and a vector model to describe the two-photon resonance. The vector model introduced by Takatsuji is an approximation which requires that the frequency difference between the input light and the intermediate states be relatively large. Recently, Brewer and $\mathrm{Hahn}^{3}$ have introduced a different vector model which is an exact solution to the three-level problem when the input frequencies are resonant with the two-photon transition frequency. The first experiments on coherent two-photon effects were performed in 1972 by Shoemaker and Brewer..$^{15}$

In this paper we show that a theory of two-photon coherent effects can be used to derive and explain two-photon resonantly enhanced parametric generation processes. Previously, these nonlinear mixing processes were described using time-dependent perturbation theory. By applying the adiabatic following (AF) solution to the vector model of Takatsuji, we show that the usual results for the two-photon resonantly enhanced nonlinear susceptibilities correspond to the $\overrightarrow{\mathrm{r}}$ vector of Feynman, Vernon, and Hellwarth, ${ }^{1}$ adiabatically following the $\vec{\gamma}$ vector in the small-angle limit. This situation allows us to make the natural extension of the theory to large angles to cover the high-power situation as the two-photon resonance is approached. The resulting AF nonlinear susceptibilities are power dependent, and the AF susceptibilities are equal to the power-independent results obtain- 
ed from perturbation theory, ${ }^{9,16,17}$ multiplied by the simple power factor $|\delta / \gamma|$. This power factor has a simple physical interpretation as the ratio of the frequency offset $\delta$ from the two-photon resonance to the precession frequency $\gamma$ of the $\vec{r}$ vector about the $\vec{\gamma}$ vector. Also, the AF solution gives an out-of-phase term at the fundamental which is responsible for energy exchange and pulse reshaping.

From our study of the vector model, we conclude that some of the previous work on two-photon coherent effects has often neglected three important effects. ${ }^{2,14,18^{-22}}$ First, third-harmonic generation and nonlinear mixing can be very important to propagation effects for linearly polarized light when the intermediate states are far from the input frequencies. For this situation, the component of the polarization, for example, at the third harmonic can be as large as the component at the fundamental. Second, there are two in-phase components of the polarization at the fundamental proportional to the populations in the two-photon ground and excited states. These terms can cause strong self-phase modulation. Third, coherent propagation effects involve large transfers of population to the two-photon excited state. In particular, for two-photon self-induced transparency, all the atoms can be in the excited state at the peak of the $2 \pi$ pulse, where the $\overrightarrow{\mathbf{r}}$ vector has been swept $\pi$ radians from its initial position. Consequently, if there are intermediate states of lower energy than the two-photon excited state, large gains may be present for lasing to these intermediate states. This point has also been mentioned by Nakatsuka et al..$^{23}$ in connection with their recent experimental work on two-photon effects.

Using the AF results for the polarization, we also study the propagation of pulses nearly resonant with a two-photon transition. It is possible to obtain pulse reshaping under these conditions, and the reshaping is due to the two related effects of a nonlinear pulse velocity and self-phase modulation. By considering circularly polarized light, we eliminate the first difficulty of nonlinear mixing and third-harmonic generation cited above. Also, by including in the analysis the polarization components cited as the second difficulty, the propagation problem is simplified, and an analytic result relating the pulse reshaping and the selfphase modulation is obtained. We present pulse propagation through $\mathrm{Rb}$ vapor as an illustrative example, and a numerical solution is given. Finally, because for this example the intermediate state is below the two-photon excited state, we discuss the problem of lasing from the excited state to the intermediate state.

The paper is organized in the following way. In Secs. II A and II B, a theory of the two-photon reso- nance is reviewed, where we attempt to bring out the important physical ideas of the formulation as well as the limitations. The theoretical approach of Hartmann ${ }^{13}$ is outlined in Sec. II A, and the alternative method of Belenov and Poluektov, ${ }^{14}$ and Takatsuji ${ }^{2,21}$ is presented in Sec. II B. The differences in these two methods are explained, and it is shown that in the proper limits, identical results are obtained. A two-photon vector model is then introduced in Sec. II C in a form most analogous to the familiar one-photon model. This allows one to study the possible two-photon analogs of well-known one-photon processes. In Sec. II D, we calculate the polarization using the two-photon vector model. Three different components of the resultant polarization are identified, and their physical significance is discussed. The adiabatic following (AF) approximation is introduced in Sec. III A and applied to the two-photon vector model, and in Sec. III B the resulting polarization is calculated. Using the AF polarization, twophoton pulse propagation is studied in Sec. III C through the coupled amplitude and phase equations. As an illustrative example, the coupled equations are integrated numerically for pulse propagation in $\mathrm{Rb}$ vapor. Section IV summarizes the work. The Appendices are devoted to three important situations not treated in the text: (A) the equalfrequency case for linearly polarized light, (B) circularly polarized light, and (C) two-photon resonantly enhanced frequency up-conversion. ${ }^{12-14}$

\section{THEORY OF THE TWO-PHOTON RESONANCE}

The theoretical treatment of coherent effects associated with the two-photon resonance has been presented in several different formalis ms. ${ }^{2,3,13,14}$ The pioneering work of Hartmann ${ }^{13}$ analyzed the three-level system for the Raman echo problem. His approach was to first make a unitary transformation to a "doubly rotating frame" to eliminate the time dependence from the Hamiltonian. Then the equation of motion for the density matrix could be solved by a series of time-independent transformations. This treatment did not require the two-level system approximation, although simplification of the final formulas gives results in agreement with the two-level theory. Later, Weingarten ${ }^{24}$ in his thesis extended Hartmann's work to the two-level approximation, which is reviewed in Sec. II A.

The approach of Belenov and Poluektov ${ }^{14}$ and Takatsuji ${ }^{2,21}$ takes a different point of view, in that initially a time-dependent unitary transformation is made which immediately recasts the theory in the two-level approximation. This method of eliminating the intermediate states was developed 
by Heitler. ${ }^{25}$ The approach is valid for any number of levels and is therefore more general. In Sec. II B we will rederive these results using an intuitive method that stresses the physical approximations which are made.

Recently, Brewer and $\mathrm{Hahn}^{3}$ have introduced a different vector model which, in contrast to the approximate theories mentioned above, is an exact solution to the three-level problem. This theory is in many ways complementary to the approximate two-photon vector model of Takatsuji that is utilized here. For the vector model of Takatsuji, the frequency of the incident light must be relatively far from resonance with respect to the single-photon transitions to the intermediate states, and the light must be applied adiabatically with respect to these intermediate states. However, the input frequencies can be either resonant or near-resonant with respect to the two-photon transition, and the model is valid for any number of intermediate states. On the other hand, while the vector model of Brewer and Hahn applies only to the three-level case when the input frequencies are resonant with the two-photon transition, there is no restriction with respect to resonance with the intermediate state and no restriction on whether the light is applied adiabatically or nonadiabatically with respect to the intermediate state.

Sections II A and B are logically independent, and according to the preferences of the reader either method can be used. The following introduction applies equally well to both methods as well as the formulation of the vector model of Sec. II C and the method of calculation of the polarization presented in Sec. II D.

The Hamiltonian describing the atom and the interaction between the atom and the applied light is assumed to be of the form

$$
\mathfrak{H C}=\mathfrak{H}_{0}-\overrightarrow{\mathrm{p}} \cdot \overrightarrow{\mathrm{E}},
$$

where $\mathcal{H}_{0}$ is the atomic Hamiltonian in the absence of the light, $\vec{p}$ is the electric-dipole-moment operator, and $\vec{E}$ is the electric field of the light. The atom is assumed to be irradiated by two linearly polarized light beams with frequencies $\omega_{1}$ and $\omega_{2}$, electric field strengths $\mathcal{E}_{1}$ and $\mathcal{E}_{2}$, and propagation vectors $k_{1}$ and $k_{2}$, respectively. Hence, the total electric field $\overrightarrow{\mathrm{E}}$ is given by

$$
\overrightarrow{\mathrm{E}}=\hat{x}\left(\mathcal{E}_{1} \cos \Phi_{1}+\mathcal{E}_{2} \cos \Phi_{2}\right),
$$

with

$$
\begin{aligned}
& \Phi_{1}=\omega_{1} t+\phi_{1}-k_{1} z, \\
& \Phi_{2}=\omega_{2} t+\phi_{2}-k_{2} z,
\end{aligned}
$$

where $\mathcal{E}_{1}, \mathcal{E}_{2}, \phi_{1}$, and $\phi_{2}$ are slowly varying in time. The case $\omega_{1}=\omega_{2}$ is treated in Appendix A.
Because the light is linearly polarized along the $x$ direction, we consider only $\pi$ transitions, and the operator $\vec{p}$ will have the form

$$
\overrightarrow{\mathrm{p}}=\hat{x} p_{x} \text {. }
$$

The case of circularly polarized light is treated in Appendix B.

We are concerned with the two-photon transition between the excited state $|1\rangle$ and the ground state $|2\rangle$ of the unperturbed Hamiltonian $\mathfrak{H}_{0}$. The matrix element of $\vec{p}$ between states $|1\rangle$ and $|2\rangle$ is zero. However, as shown in Fig. 1, there are usually many (and at least one) other atomic states which are connected by dipole transitions to the two-photon resonant states $|1\rangle$ and $|2\rangle$ under study. We assume that $\omega_{1}$ and $\omega_{2}$ are not resonant with any transition frequency but that the sum $\omega_{1}+\omega_{2}$ is approximately equal to the transition frequency $\Omega_{12}$ between states $|1\rangle$ and $|2\rangle$.

\section{A. Three-level system}

The most simple system of interest for two-photon or Raman-type resonances is the three-level system. This system was analyzed for circularly polarized light in a very elegant manner by Hart$\operatorname{mann}^{13}$; here we adapt his approach to linearly polarized light. The Hamiltonian [Eq. (1)] for the three levels is written in matrix form as the following:

$$
\mathcal{H}=\left[\begin{array}{ccc}
\hbar \Omega_{1} & 0 & -\frac{1}{2} p_{1 n} \mathcal{E}_{1} e^{-i \Phi_{1}} \\
0 & \hbar \Omega_{2} & -\frac{1}{2} p_{2 n} \mathcal{E}_{2} e^{i \Phi_{2}} \\
-\frac{1}{2} p_{n 1} \mathcal{E}_{1} e^{i \Phi_{1}} & -\frac{1}{2} p_{n 2} \mathcal{E}_{2} e^{-i \Phi_{2}} & \hbar \Omega_{n}
\end{array}\right],
$$

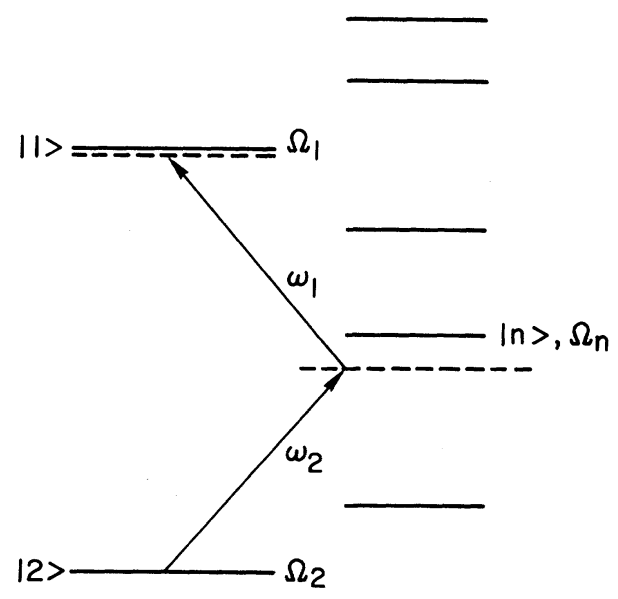

FIG. 1. Generalized energy-level diagram for the twophoton transition between states $|1\rangle$ and $|2\rangle$. 
where

$$
p_{m n}=\left\langle m\left|p_{x}\right| n\right\rangle \text {. }
$$

The equation of motion for the density matrix $\rho$ is given by

$$
\frac{\partial \rho}{\partial t}=\frac{i}{\hbar}[\rho, \mathfrak{H}]
$$

This simple form for the Hamiltonian, in which the off-diagonal terms have been limited to coupling with one field each, can be justified if the intermediate state $|n\rangle$ is nearly resonant. This form allows a tremendous simplification in the theory. The more general case in which coupling to both fields including the counter-rotating components of these fields, as well as the inclusion of other intermediate states, is more easily treated with the alternative approach outlined in Sec. II B.

The problem can be further simplified by transforming to a "doubly rotating" frame which removes the explicit time dependence from the Hamiltonian. This transformation is accomplished by the unitary operator

$$
U_{1}=e^{-i \Lambda}
$$

where

$$
\Lambda=\left[\begin{array}{ccc}
-\Phi_{1} & 0 & 0 \\
0 & +\Phi_{2} & 0 \\
0 & 0 & 0
\end{array}\right]
$$

Then, defining

$$
\rho_{R}=U_{1} \rho U_{1}^{-1},
$$

we obtain

$$
\frac{\partial \rho_{R}}{\partial t}=\frac{i}{\hbar}\left[\rho_{R}, \mathfrak{H}_{E}\right],
$$

where the effective Hamiltonian $\mathfrak{H}_{E}$ in this doubly rotating frame is

$$
\mathfrak{H}_{E}=U_{1} \mathfrak{H} U_{1}^{-1}+\hbar \frac{\partial \Lambda}{\partial t}
$$

or

$$
\mathcal{H}_{E}=\left[\begin{array}{ccc}
\hbar\left(\Omega_{1}-\omega_{1}^{\prime}\right) & 0 & -\frac{1}{2} p_{1 n} \mathcal{E}_{1} \\
0 & \hbar\left(\Omega_{2}+\omega_{2}^{\prime}\right) & -\frac{1}{2} p_{2 n} \mathcal{E}_{2} \\
-\frac{1}{2} p_{n 1} \mathcal{E}_{1} & -\frac{1}{2} p_{n 2} \mathcal{E}_{2} & \hbar \Omega_{n}
\end{array}\right],
$$

with

$$
\begin{aligned}
& \omega_{1}^{\prime}=\omega_{1}+\frac{\partial \phi_{1}}{\partial t}, \\
& \omega_{2}^{\prime}=\omega_{2}+\frac{\partial \phi_{2}}{\partial t} .
\end{aligned}
$$

The term $\hbar(\partial \Lambda / \partial t)$ arises because the transformation $U_{1}$ is time dependent. At this point one can continue by diagonalizing this Hamiltonian to solve the problem. This procedure was used by Hart$\operatorname{mann}^{13}$ in the Raman-echo work. Here we use a different approach, where a two-level approximation is made; the needed transformation is described below.

The Hamiltonian is of the form

$$
\mathfrak{H}_{E}=\mathfrak{H}_{E}^{0}+\lambda H,
$$

where $\lambda H$ is the off-diagonal part of $\mathfrak{H}_{E}$. The expansion parameter $\lambda$ is of the order of $\vec{p} \cdot \vec{E} / \hbar \Delta \omega$, where $\Delta \omega$ is the frequency offset of the intermediate state. We make the transformation

$$
\mathcal{H}_{E}^{\prime \prime}=U_{2} \mathcal{H}_{E} U_{2}^{-1}=\mathcal{H}_{E}^{0}+\frac{1}{2} i \lambda[H, \S]+O\left(\lambda^{3}\right),
$$

where

$$
U_{2}=e^{-i \delta},
$$

and

$$
S_{n m}=-i \lambda H_{n m} /\left(\mathcal{H}_{E_{m m}}^{0}-\mathcal{H}_{E_{n n}}^{0}\right) .
$$

If we drop terms of order $\lambda^{3}$ and smaller, the Hamiltonian is reduced to the form

$$
\mathcal{H}_{E}^{\prime \prime}=\left[\begin{array}{ccc}
\hbar\left(\Omega_{1}-\omega_{1}^{\prime}\right)+\Delta E_{1} & \frac{p_{1 n} p_{n 2} \mathcal{E}_{1} \mathcal{E}_{2}}{4 \hbar \Delta \omega} & 0 \\
\frac{p_{2 n} p_{n 1} \mathcal{E}_{1} \mathcal{E}_{2}}{4 \hbar \Delta \omega} & \hbar\left(\Omega_{2}+\omega_{2}^{\prime}\right)+\Delta E_{2} & 0 \\
0 & 0 & \hbar \Omega_{n}-\Delta E_{1}-\Delta E_{2}
\end{array}\right],
$$

where

$$
\begin{aligned}
& \frac{1}{\Delta \omega}=\frac{1}{2}\left(\frac{1}{\omega_{2}-\Omega_{n 2}}+\frac{1}{\Omega_{1 n}-\omega_{1}}\right), \\
& \Omega_{i j}=\Omega_{i}-\Omega_{j},
\end{aligned}
$$

and the Stark shifts ${ }^{26,27}$ are given by

$$
\begin{aligned}
& \Delta E_{1}=\left|p_{1 n} \mathcal{E}_{1}\right|^{2} / 4 \hbar\left(\Omega_{1 n}-\omega_{1}\right), \\
& \Delta E_{2}=\left|p_{2 n} \mathcal{E}_{2}\right|^{2} / 4 \hbar\left(\omega_{2}-\Omega_{n 2}\right) .
\end{aligned}
$$

The self-phase-modulation terms $\partial \phi_{1} / \partial t$ and $\partial \phi_{2} / \partial t$ have been deleted $\left(\omega_{1}^{\prime} \rightarrow \omega_{1}\right.$ and $\left.\omega_{2}^{\prime} \rightarrow \omega_{2}\right)$ from Eq. (18), since they would lead to terms of order high- 
er than $\lambda^{2}$.

The equation of motion for the density matrix becomes

$$
\frac{\partial \rho^{\prime \prime}}{\partial t}=\frac{i}{\hbar}\left[\rho^{\prime \prime}, \mathcal{H}_{E}^{\prime \prime}\right]
$$

where

$$
\rho^{\prime \prime}=U_{2} \rho_{R} U_{2}^{-1}
$$

By keeping terms only to order $\lambda^{2}$, i.e., $\sim[(\overrightarrow{\mathrm{p}} \cdot \overrightarrow{\mathrm{E}}) /$ $\hbar \Delta \omega]^{2}$, this transformation corresponds to a change of basis states from the states $\mathfrak{F}_{E}^{0}$ to the orthogonalized, perturbed states obtained in perturbation theory.

The unitary operator $U_{2}$ has been treated as time independent, i.e., we assume that the fields are turned on and off adiabatically. This condition of adiabaticity is satisfied if

$$
\left|\frac{\partial \mathscr{E}}{\partial t}\right| \ll|\Delta \omega \mathcal{E}| \text {. }
$$

The single-photon adiabatic requirement of Eq. (23) is a basic limitation to the two-level approximation and the associated vector model. However, one can usually choose the frequencies of the fields involved to satisfy this requirement by making the mistuning from the intermediate-state resonance sufficiently large. The time-dependent perturbation-theory calculations ${ }^{9,16,17}$ of the nonlinear mixing coefficients make this same approximation.

The intermediate state in the Hamiltonian of Eq. (17) is completely decoupled from the other states and therefore can be dropped from consideration. This reduces the Hamiltonian to $2 \times 2$ form consisting only of the upper left corner of the matrix (17); hence, we need only retain a $2 \times 2$ density matrix. Consequently, Eq. (21) can be considered as a $2 \times 2$ matrix equation, and as a result the vector model for two-level systems can be used directly to derive equations of motion for this system (see Sec. II C). Note that the Stark shifts of the states $|1\rangle$ and $|2\rangle$ have appeared in a natural way, and that the twophoton transition probability can be obtained in the usual manner by substituting the square of absolute value of the off-diagonal matrix element connecting these states into Fermi's golden rule.

\section{B. Multi-level systems}

In order to take into account the presence of more than one intermediate state, it is convenient to use the method of Belenov and Poluektov ${ }^{14}$ and Takatsuji. $^{2,21}$ This treatment also allows us to consider the coupling of both fields in each offdiagonal matrix element. In this section we rederive these earlier results using an intuitive method that stresses the physical approximations which are made. Our approach is most closely related to that recently used by Takatsuji, ${ }^{21}$ except that we are using the Schrödinger-picture and the density-matrix formalism of spin-resonance and single-photon coherent optics. The calculation follows in a manner quite similar to that used on the three-level system except that the transformation to a rotating frame is made last instead of first. The physical significance of the first transformation is the following: Initially, there is no matrix element of the electric dipole moment between the resonant two-photon states designated as $|1\rangle$ and $|2\rangle$. However, the application of the near-resonant light changes these original states into the new states $|1\rangle^{\prime}$ and $|2\rangle^{\prime}$, which include a linear superposition of many of the original unperturbed states $|n\rangle$. Once the states $|1\rangle^{\prime}$ and $|2\rangle^{\prime}$ have been calculated, the problem can be recast in terms of these two states. This is possible because now there is a matrix element of the electric dipole moment between states $|1\rangle^{\prime}$ and $|2\rangle^{\prime}$ and the light is nearly resonant to the transition frequency between these states.

The unitary operator $U$ that transforms to the primed basis states can be written as

$$
U=e^{-i S}=1-i S-\frac{1}{2} S S+\cdots,
$$

where $S$ is a Hermitian matrix with no diagonal elements and is given by

$$
\begin{aligned}
S_{n i}=\frac{-i p_{n i}}{2 \hbar}[ & \mathcal{E}_{1}\left(\frac{e^{-i \Phi_{1}}}{\Omega_{n i}-\omega_{1}}+\frac{e^{+i \Phi_{1}}}{\Omega_{n i}+\omega_{1}}\right) \\
& \left.+\mathcal{E}_{2}\left(\frac{e^{-i \Phi_{2}}}{\Omega_{n i}-\omega_{2}}+\frac{e^{+i \Phi_{2}}}{\Omega_{n i}+\omega_{2}}\right)\right] .
\end{aligned}
$$

The normalized and orthogonal basis states $|i\rangle^{\prime}$ are given by ${ }^{28}$

$$
|i\rangle^{\prime}=\sum_{n} U_{n i}^{-1}|n\rangle
$$

It is important to notice that, to first order in $S$, the new states thus obtained are the same as those calculated by conventional first-order time-dependent perturbation theory. The usual time-independent terms are omitted in Eq. (25) because the interaction (light beams) is turned on adiabatically. ${ }^{29}$

The equation of motion for the density matrix $\rho$ in terms of the original unperturbed states of $\mathcal{H}_{0}$ is

$$
\frac{\partial \rho}{\partial t}=\frac{i}{\hbar}[\rho, \mathfrak{H}] .
$$

If we define

$$
\rho^{\prime}=U \rho U^{-1},
$$


then Eq. (27) can be rewritten in terms of an effective Hamiltonian $\mathfrak{H}_{e}^{\prime}$ in the primed representation as

$$
\frac{\partial \rho^{\prime}}{\partial t}=\frac{i}{\hbar}\left[\rho^{\prime}, \mathcal{H}_{e}^{\prime}\right],
$$

with

$$
\mathfrak{H}_{e}^{\prime}=U \mathfrak{H} U^{-1}+\hbar \frac{\partial S}{\partial t}-\frac{\hbar}{2 i}\left[\frac{\partial S}{\partial t}, S\right]
$$

to second order in $S$. In Eq. (30) the additional terms are present because the transformation is time dependent. The relevant matrix elements of $\mathfrak{H C}_{e}^{\prime}$ are

$$
\begin{aligned}
& \mathcal{H}_{e_{21}}^{\prime}=\mathcal{H}_{e_{12}^{\prime *},}^{*^{*}} \\
& \mathcal{H}_{e_{11}}^{\prime}=\hbar \Omega_{1}+\Delta E_{1}, \\
& \mathcal{H}_{e_{22}}^{\prime}=\hbar \Omega_{2}+\Delta E_{2},
\end{aligned}
$$

where only the stationary terms are kept for the diagonal elements. To second order, the off-diago- nal element $\mathfrak{H}_{e_{12}}^{\prime}$ is given by

$$
\begin{aligned}
& \mathcal{H}_{e_{12}}^{\prime}=\sum_{n}\left\{\frac{1}{2} \hbar\left(\Omega_{n 1}+\Omega_{n 2}\right) S_{1 n} S_{n 2}-i S_{1 n} \mathcal{H}_{n 2}+i \mathcal{C}_{1 n} S_{n 2}\right. \\
&\left.-\frac{\hbar}{2 i}\left[\left(\frac{\partial S_{1 n}}{\partial t}\right) S_{n 2}-S_{1 n}\left(\frac{\partial S_{n 2}}{\partial t}\right)\right]\right\}
\end{aligned}
$$

The optical Stark shift ${ }^{26,27}$ of any state $|i\rangle^{\prime}$ is designated by $\Delta E_{i}$, where

$$
\Delta E_{i}=-\frac{1}{4}\left[\chi_{i}\left(\omega_{1}\right) \mathcal{E}_{1}^{2}+\chi_{i}\left(\omega_{2}\right) \mathcal{E}_{2}^{2}\right] .
$$

The symbol $\chi_{i}\left(\omega_{1}\right)$ represents the linear electric susceptibility for linearly polarized light of angular frequency $\omega_{1}$ of a single atom in state $|i\rangle$ and is given explicitly by

$$
\chi_{i}\left(\omega_{1}\right)=\sum_{n} \frac{p_{i n} p_{n i} 2 \Omega_{n i}}{\hbar\left(\Omega_{n i}^{2}-\omega_{1}^{2}\right)} .
$$

Making the counter-rotating-wave approximation, we can evaluate Eq. (32) as

$$
\begin{aligned}
\mathcal{F}_{e_{12}}^{\prime}=-\sum_{n}\left\{\frac{p_{1 n} p_{n 2}\left(\Omega_{n 1}+\Omega_{n 2}\right)}{8 \hbar}[\right. & \frac{\mathcal{E}_{1}^{2} e^{-i 2 \Phi_{1}}}{\left(\Omega_{n 1}+\omega_{1}\right)\left(\Omega_{n 2}-\omega_{1}\right)}+\frac{\mathcal{E}_{2}^{2} e^{-i 2 \Phi_{2}}}{\left(\Omega_{n 1}+\omega_{2}\right)\left(\Omega_{n 2}-\omega_{2}\right)} \\
& +\mathcal{E}_{1} \mathcal{E}_{2} e^{-i\left(\Phi_{1}+\Phi_{2}\right)}\left(\frac{1}{\left(\Omega_{n 1}+\omega_{1}\right)\left(\Omega_{n 2}-\omega_{1}\right)}+\frac{1}{\left(\Omega_{n 1}+\omega_{2}\right)\left(\Omega_{n 2}-\omega_{2}\right)}\right) \\
& \left.\left.+\mathcal{E}_{1} \mathcal{E}_{2} e^{-i\left(\Phi_{1}-\Phi_{2}\right)}\left(\frac{1}{\left(\Omega_{n 1}+\omega_{1}\right)\left(\Omega_{n 2}-\omega_{1}\right)}+\frac{1}{\left(\Omega_{n 1}-\omega_{2}\right)\left(\Omega_{n 2}+\omega_{2}\right)}\right)\right]\right\}
\end{aligned}
$$

The off-diagonal matrix element $\mathcal{H}_{e_{12}}^{\prime}$ has terms oscillating at the frequencies $2 \omega_{1}, 2 \omega_{2}, \omega_{1}+\omega_{2}$, and $\omega_{1}-\omega_{2}$. The first two will result in two-photon transitions in which both photons have equal frequency, the third results in two-photon transitions with unequal-frequency photons, and the final term corresponds to Raman-type transitions. We shall assume that one of these four frequencies is nearly resonant to the transition frequency $\left(\mathcal{H}^{\prime} e_{1}\right.$ $\left.-\mathcal{F}_{e_{22}}^{\prime}\right) / \hbar$ between the states $|1\rangle^{\prime}$ and $|2\rangle^{\prime}$, and that all other transitions can be neglected. Thus, when such resonance occurs, Eq. (29) can be considered as a $2 \times 2$ matrix equation describing the time dependence of the density matrix restricted to the mixed states $|1\rangle^{\prime}$ and $|2\rangle^{\prime}$.

For our subsequent development of the problem we choose the case $\omega_{1}+\omega_{2} \simeq \Omega_{12}$. The case $\omega_{1}-\omega_{2}$ $\simeq \Omega_{12}$ is similar and only involves a change in sign whenever $\omega_{2}$ appears. The case $2 \omega_{1} \simeq \Omega_{12}$ is treated in Appendix A. With this assumption, only the term involving $e^{-i\left(\Phi_{1}+\Phi_{2}\right)}$ in $\mathcal{J C}_{e_{12}^{\prime}}^{\prime}$ need be retained, and we can write

$$
\mathcal{H}_{e_{12}}^{\prime}=-\left|\mathcal{H}_{e_{12}^{\prime}}^{\prime}\right| e^{-i\left(\Phi_{1}+\Phi_{2}+\beta\right)} .
$$

We now transform into the rotating frame, where the Hamiltonian is no longer oscillatory and where the matrix elements are real, by the unitary trans formation $R$ :

$$
R=\left[\begin{array}{cc}
e^{i\left(\Phi_{1}+\Phi_{2}+\beta\right) / 2} & 0 \\
0 & e^{-i\left(\Phi_{1}+\Phi 2^{+\beta}\right) / 2}
\end{array}\right] .
$$

Following the same procedure as before, we define

$$
\rho^{\prime \prime}=R \rho^{\prime} R^{-1},
$$

and the equation of motion for the density matrix in the double-primed representation becomes

$$
\frac{\partial \rho^{\prime \prime}}{\partial t}=\frac{i}{\hbar}\left[\rho^{\prime \prime}, \mathfrak{H}_{E}^{\prime \prime}\right]
$$

with the new effective Hamiltonian $\mathfrak{H}_{E}^{\prime \prime}$ equal to 


$$
\mathcal{H}_{E}^{\prime \prime}=R \mathcal{H}_{e}^{\prime \prime} R^{-1}-\frac{\hbar}{i} \frac{\partial R}{\partial t} R^{-1}
$$

Again, the second term in Eq. (40) is due to the time dependence of the transformation $R$. $\mathfrak{H}_{E}^{\prime \prime}$ is displayed as

$$
\mathcal{H}_{E}^{\prime \prime}=\left[\begin{array}{cc}
\mathcal{H}_{e_{11}}^{\prime}-\frac{1}{2} \hbar\left(\omega_{1}^{\prime}+\omega_{2}^{\prime}\right) & -\left|\mathcal{H}_{e_{12}}^{\prime}\right| \\
-\left|\mathcal{H}_{e_{12}}^{\prime}\right| & \mathcal{H}_{e_{22}}^{\prime}+\frac{1}{2} \hbar\left(\omega_{1}^{\prime}+\omega_{2}^{\prime}\right)
\end{array}\right] .
$$

The Hamiltonian $\mathcal{H}_{E}^{\prime \prime}$ is now in a form most suitable for the vector model since all the matrix elements are real. It is easily shown that the Hamiltonian of Eq. (17) can be brought to the same form as above by transforming to the frame where the offdiagonal elements are real and by shifting the zero-point reference energy, i.e., adding the factor $\frac{1}{2} \hbar\left(\omega_{1}^{\prime}+\omega_{2}^{\prime}\right)$ to the diagonal elements.

\section{The vector model}

Equation (21) of Sec. II A and Eq. (39) of Sec. II B, which describe the time dependence of the density matrix $\rho^{\prime \prime}$, can now be expressed in terms of the vector model of Ref. 1. As shown in Fig. 2, the unit vector $\overrightarrow{\mathbf{r}}$ precesses about the $\vec{\gamma}$ vector $(\vec{\gamma}=\vec{\omega}$ of Ref. 1), i.e.,

$$
\partial \overrightarrow{\mathrm{r}} / \partial t=\vec{\gamma} \times \overrightarrow{\mathrm{r}} .
$$

The equations of motion for the components of $\vec{r}$ are given by

$$
\begin{aligned}
& \partial r_{1} / \partial t=-\gamma_{3} r_{2}, \\
& \partial r_{2} / \partial t=-\gamma_{1} r_{3}+\gamma_{3} r_{1}, \\
& \partial r_{3} / \partial t=+\gamma_{1} r_{2} .
\end{aligned}
$$

The components of $\vec{r}$ are given in terms of the density matrix by

$$
\begin{aligned}
& r_{1}=\rho_{12}^{\prime \prime}+\rho_{21}^{\prime \prime}, \\
& r_{2}=i\left(\rho_{12}^{\prime \prime}-\rho_{21}^{\prime \prime}\right), \\
& r_{3}=\rho_{11}^{\prime \prime}-\rho_{22}^{\prime \prime},
\end{aligned}
$$

and the components of $\vec{\gamma}$ are given in terms of the effective Hamiltonian by

$$
\begin{aligned}
& \gamma_{1}=\left(\mathcal{H}_{E_{12}}^{\prime \prime}+\mathcal{H}_{E_{21}}^{\prime \prime}\right) / \hbar, \\
& \gamma_{2}=i\left(\mathcal{H}_{E_{12}}^{\prime \prime}-\mathcal{H}_{E_{21}}^{\prime \prime}\right) / \hbar, \\
& \gamma_{3}=\left(\mathcal{H C}_{E_{11}^{\prime \prime}}^{\prime \prime}-\mathcal{H}_{E_{22}}^{\prime \prime}\right) / \hbar .
\end{aligned}
$$

For the case of two-photon transitions with unequal frequency fields the explicit expression for $\vec{\gamma}$ can be written as

$$
\begin{aligned}
& \gamma_{1}=-\kappa \mathcal{E}_{1} \mathcal{E}_{2}, \\
& \gamma_{2}=0, \\
& \gamma_{3}=\Omega_{12}-\left(\omega_{1}^{\prime}+\omega_{2}^{\prime}\right)+\left(\Delta E_{1}-\Delta E_{2}\right) / \hbar,
\end{aligned}
$$

where $\kappa$ is the two-photon gyroelectric ratio, defined as

$$
\kappa=\frac{1}{2 \hbar^{2}}\left|\sum_{n} p_{1^{n}} p_{n 2}\left(\frac{1}{\Omega_{n 2}-\omega_{1}}+\frac{1}{\Omega_{n 2}-\omega_{2}}\right)\right| .
$$

Thus, $\left|\gamma_{1}\right|$ is the two-photon Rabi precession frequency; the angular-frequency offset from the twophoton resonance, including the Stark shift, is given by $\gamma_{3}$. The definition for the $\vec{r}$ vector [Eqs. (44)] is the same as the one-photon vector model. In particular, the component $r_{3}$ has the usual interpretation as the difference in the population probabilities of the states $|1\rangle^{\prime}$ and $|2\rangle^{\prime}$.

This formal similarity between the one-photon and two-photon vector models has important consequences. Many one-photon coherent processes can be adequately described by the vector model alone. It is important to notice that, even though the two-photon vector model assumes that the light was applied adiabatically with respect to the single-photon transitions to the intermediate states, the vector model is valid for nonadiabatic two-photon processes. Processes such as photon echoes, ${ }^{30}$ optical nutation, ${ }^{31}$ adiabatic following, ${ }^{4-8}$ and adiabatic rapid passage,${ }^{32-34}$ clearly will have their two-photon analogs. In fact, the conditions for these processes, expressed in terms of the vectors $\vec{\gamma}$ and $\overrightarrow{\mathrm{r}}$, are identical for the one- and two-photon processes. Of course, the expressions for $\vec{\gamma}$ are different for the two cases. For example, the Stark-shift term in $\gamma_{3}$ is not present in the one-photon case. Thus, generally speaking, the physical interpretations of the two-photon effects will be more complicated. For coherentpropagation effects such as self-induced transparency, ${ }^{35}$ the vector model must be coupled with the Maxwell equations. For the two-photon case, two optical fields are coupled with the vector mod-

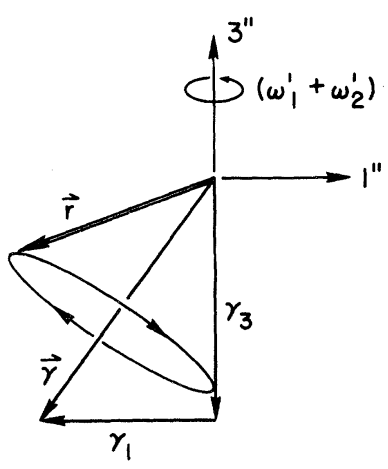

FIG. 2. Precession of the $\vec{r}$ vector about the $\vec{\gamma}$ vector in the double-primed reference frame (rotating-coordinate frame). 
el. Because of the presence of the additional field and the optical Stark shift, it is difficult to ascertain whether a given one-photon, coherent, propagation effect will have its two-photon analog.

We should point out that while, in general, twophoton coherent effects are more complicated than one-photon effects, they are correspondingly richer. One can therefore look for two-photon effects that have no one-photon analogs. One such effect, self-induced adiabatic rapid passage, has recently been proposed..$^{36}$

\section{Calculation of the polarization}

The polarization is uniquely determined by the components of $\vec{r}$. However, the intuitive picture for the polarization given by the vector model for the one-photon case does not apply to the two-photon case, and the polarization must be formally calculated by transformation techniques.

Given the $\overrightarrow{\mathbf{r}}$ components, we have the density matrix $\rho^{\prime \prime}$ in the double primed representation as

$$
\rho^{\prime \prime}(t)=\frac{1}{2}\left[\begin{array}{cc}
1+r_{3} & r_{1}-i r_{2} \\
r_{1}+i r_{2} & 1-r_{3}
\end{array}\right]
$$

and the density matrix $\rho$, in the "laboratory frame" and in terms of the original unperturbed states of $\mathfrak{H}_{0}$, is easily obtained by the reverse series of transformations for Sec. II A

$$
\rho(t)=U_{1}^{-1} U_{2}^{-1} \rho^{\prime \prime}(t) U_{2} U_{1},
$$

and for Sec. II B

$$
\rho(t) \equiv U^{-1} R^{-1} \rho^{\prime \prime}(t) R U .
$$

Then, the polarization for a single atom can be calculated for the case of unequal frequency fields as

$$
\overrightarrow{\mathrm{p}}(t)=\operatorname{Tr} \overrightarrow{\mathrm{p}} \rho(t)=\hat{x} p(t),
$$

where

$$
p(t)=p_{\mathrm{I}}(t)+p_{\mathrm{II}}(t)+p_{\mathrm{III}}(t) .
$$

The three different contributions to the polarization are

$$
\begin{aligned}
p_{\mathrm{I}}(t)= & \frac{1}{2}\left[\chi_{1}\left(\omega_{1}\right)\left(1+r_{3}\right)+\chi_{2}\left(\omega_{1}\right)\left(1-r_{3}\right)\right] \mathcal{E}_{1} \cos \Phi_{1} \\
& +\frac{1}{2}\left[\chi_{1}\left(\omega_{2}\right)\left(1+r_{3}\right)+\chi_{2}\left(\omega_{2}\right)\left(1-r_{3}\right)\right] \mathcal{E}_{2} \cos \Phi_{2},
\end{aligned}
$$

$$
p_{\text {II }}(t)=\operatorname{Re} \hbar \kappa\left(r_{1}-i r_{2}\right)\left(\mathcal{E}_{2} e^{-i \Phi_{1}}+\mathcal{E}_{1} e^{-i \Phi_{2}}\right),
$$

and

$$
p_{\text {III }}(t)=\operatorname{Re}\left(r_{1}-i r_{2}\right) \sum_{n}\left\{\frac{p_{2 n} \dot{p}_{n 1}}{2 \hbar}\left[\left(\frac{1}{\Omega_{n 2}+\omega_{1}}+\frac{1}{\Omega_{n 1}-\omega_{1}}\right) \mathcal{E}_{1} e^{-i\left(2 \Phi_{1}+\Phi_{2}+\beta\right)}+\left(\frac{1}{\Omega_{n 2}+\omega_{2}}+\frac{1}{\Omega_{n 1}-\omega_{2}}\right) \mathcal{E}_{2} e^{-i\left(\Phi_{1}+2 \Phi_{2}+\beta\right)}\right]\right\}
$$

From an inspection of Eqs. (51)-(55) several observations can be made. The $p_{\mathrm{I}}$ components give a polarization at the driving frequencies. These components are due to the allowed onephoton transitions $|1\rangle \rightarrow|n\rangle$ and $|2\rangle \rightarrow|n\rangle$, and are proportional to the population of the states $|1\rangle$ and $|2\rangle$. These terms can cause self-focusing, self-defocusing, and self-phase modulation of the input waves $\vec{E}_{1}$ and $\vec{E}_{2}$. Surprisingly, as shown in Sec. III, the inclusion of the $p_{\mathrm{I}}$ components actually simplifies the propagation problem for the AF model and allows for an analytic solution which relates the pulse reshaping and the selfphase modulation.

The $p_{\text {II }}$ components are in close analogy with the one-photon vector model in that these components of the polarization are at the frequencies of the driving fields and are simply proportional to the transverse components of the $\overrightarrow{\mathbf{r}}$ vector. However, the terms show an interesting interchange of fields which is characteristic of the two-photon resonance. The out-of-phase term proportional to $r_{2}$ determines the energy exchange between the atoms and the propagating waves and is responsible for slow pulse velocities and pulse reshaping. If a transverse relaxation time $T_{2}$ is introduced into the Bloch equations (43), a new component of the out-of-phase term is introduced which gives the usual two-photon absorption. The in-phase component of $p_{I I}$ is analogous to its counterpart in onephoton processes, and it can cause important effects such as self-focusing, self-defocusing, and self-phase modulation. The importance of this term in pulse propagation will be clearly demonstrated in the example given in Sec. III.

The $p_{\text {III }}$ components are responsible for the twophoton resonantly enhanced generation of mixed frequencies. It is interesting to note that these components do not appear when one uses the threelevel approximations of Sec. II A, and they have generally been neglected in the previous theories of coherent two-photon propagation phenomena. The mixed-frequency terms do not arise in the three-level approximation because the simplified Hamiltonian [Eq. (4)] assumes that each input field is near resonant to only one transition and then couples only one field in each off-diagonal element. The more general treatment in Sec. II B shows that 
$p_{\text {III }}$ can be comparable in magnitude with $p_{\mathrm{I}}$ and $p_{\text {II }}$ and must be treated properly to describe twophoton effects in general. One important result of our calculation, as shown in Appendix B, is that for circularly polarized input light, $p_{\mathrm{III}}=0$. For future two-photon resonance experiments, the use of circularly polarized light is obviously advisable. If linearly polarized light must be used, it is possible to make $p_{\text {III }}$ negligible compared to $p_{\text {II }}$ by using resonant enhancement where $\Omega_{n_{2}}$ is very close to $\omega_{2}$. For the study of local effects (photon echoes, optical nutation, adiabatic rapid passage, and optical Stark shifts) this is a viable procedure, but it cannot generally be applied to the study of propagation problems, as we will show in Sec. III C.

\section{THE ADIABATIC FOLLOWING APPROXIMATION}

For a single-photon transition, the adiabatic following approximation has been shown, both experimentally and theoretically, ${ }^{4-8}$ to rather accurately describe the propagation of near-resonant light. By "near-resonant" it is meant that the frequency difference between the input light and the resonance line is very small compared to the resonant frequency, but large compared to the linewidth. This model bridges the gap between the region where linear dispersion theory is applicable and the region where the entire set of Bloch equations must be coupled to the wave equation. The approximation corresponds to the situation where the atomic pseudomoments remain aligned along the changing effective field of the laser light, and an immediate solution to the point-response problem is obtained. The near-resonant atomic response is expressed as a nonlinear electric susceptibility, allowing for direct comparison with other nonlinear mechanisms and for convenient numerical integration of the coupled Maxwell-Bloch equations.

As was shown in Sec. II, the interaction between atoms and light nearly resonant with a two-photon transition can be described by the familiar vector model of spin resonance and single-photon coherent optics. Thus, the adiabatic following approximation can also be applied to the two-photon transition to achieve a convenient and important simplification of the two-photon problem. For this case the $\overrightarrow{\mathbf{r}}$ vector is aligned along the $\gamma$ vector, and a nonlinear susceptibility is again obtained which describes the dielectric response of the atom to the near-resonant light. For the situation where the angle between $\vec{\gamma}$ and the axis of rotation is small, the nonlinear susceptibility is the same as that obtained from time-dependent perturbation theory. However, the AF solution applies to large angles as well, and the power de- pendence of the susceptibility is thereby obtained. Also, the AF solution gives an out-of-phase term which is responsible for energy exchange and pulse reshaping.

\section{A. The vector-model solution}

The AF approximation is illustrated in Fig. 3, from which the solutions for $r_{1}$ and $r_{3}$ are immediately obtained as

$$
r_{1}=( \pm) \sin \theta
$$

and

$$
r_{3}=(\mp) \cos \theta,
$$

where $\theta$ is the angle between $\vec{\gamma}$ and the $3^{\prime \prime}$ axis. The above AF solutions for $r_{1}$ and $r_{3}$ can be rewritten as

$$
r_{1}=(\mp) \gamma_{1} / \gamma
$$

and

$$
r_{3}=(\mp) \gamma_{3} / \gamma,
$$

with

$$
\gamma=+\left(\gamma_{3}^{2}+\gamma_{1}^{2}\right)^{1 / 2}
$$

and $\gamma_{1}$ and $\gamma_{3}$ are given by Eq. (46). The upper sign is used in Eqs. (56)-(59) and subsequent formulas when the initial $\gamma_{3}$ is positive, i.e., when $\gamma_{3}(z=0, t=-\infty)>0$, and conversely, the lower sign is used when $\gamma_{3}(0,-\infty)<0$. For $\gamma_{3}(0,-\infty)>0$, $\overrightarrow{\mathrm{r}}$ is antiparallel to $\vec{\gamma}$, while, for $\gamma_{3}(0,-\infty)<0, \overrightarrow{\mathrm{r}}$ is parallel to $\vec{\gamma}$ as shown in Fig.3. As defined, $\gamma_{1}$ is always negative.

Equations (58)-(60) are not in the usual AF form, ${ }^{4-8}$ but they have been written in the above manner to allow for adiabatic-passage effects (inversion), if the frequency is swept through the resonance. For example, it has been shown that optical Stark shifts in $\gamma_{3}$ can sweep the offset through the line and invert the two-photon resonance. ${ }^{36}$

The $r_{2}$ component is very small compared to $r_{1}$ and $r_{3}$, and can be obtained from $r_{1}$ by Eq. (43a) as

$$
r_{2}=-\frac{1}{\gamma_{3}} \frac{\partial r_{1}}{\partial t}
$$

The above two-photon AF solutions are valid as long as the three AF conditions are satisfied. These conditions have been given earlier ${ }^{8}$ but will be restated here for completeness. It is important to realize that these conditions are in addition to the one-photon adiabatic requirement of Eqs. (23) and (25), which is needed for the two-level approximation to be valid. Conditions I and II are the same as those of spin resonance and single-photon coherent optics for the adiabatic rapid passage. 


\section{ADIABATIC FOLLOWING}

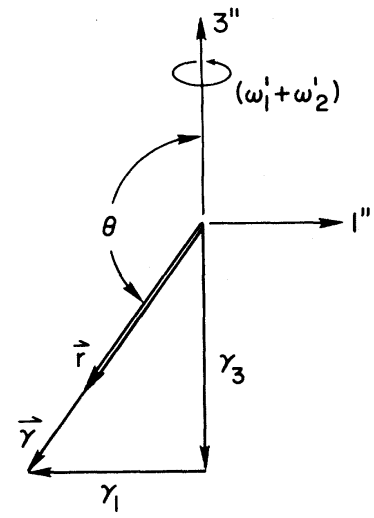

FIG. 3. The two-photon adiabatic following approximation, where the $\overrightarrow{\mathbf{r}}$ vector remains closely aligned along the $\vec{\gamma}$ vector. Here, $\gamma_{3}<0, \gamma_{1}<0$, and the diagram is shown in the double-primed reference frame (rotatingcoordinate frame).

Condition I states that the direction of the $\overrightarrow{\mathrm{r}}$ vector should change slowly compared to the precession frequency $\gamma$ of the $\vec{r}$ vector about $\vec{\gamma}$. Expressed mathematically this becomes

$$
|\partial \theta / \partial t| \ll \gamma \text {. }
$$

Condition II requires that the incoherently absorbed energy per unit volume is very small compared to the saturated energy capacity per unit volume of the system. This saturation energy capacity $U_{s}$ is given by

$$
U_{s}=\frac{1}{2} \hbar \Omega_{12} N,
$$

where $N$ is the atomic number density. Condition III is unique to AF and comes from the additional requirement that the angle between $\overrightarrow{\mathrm{r}}$ and $\vec{\gamma}$ be very much smaller than the angle between $\vec{\gamma}$ and the $3^{\prime \prime}$ axis. This implies that

$$
\left|r_{2}\right| \ll\left|r_{1}\right|
$$

which is condition III. If these three conditions are satisfied, the AF solution is a very good approximate solution to the Bloch equations (43). Crisp has studied the validity of the AF approximation by means of a novel approximation scheme ${ }^{37}$ and his results are in agreement with the above relatively simple discussion.

\section{B. Nonlinear polarization}

The adiabatic following solution allows us to immediately calculate the atomic polarization. The polarization is obtained by simply inserting the AF solutions [Eqs. (58)-(61)] into the expression for the polarization [Eq. (52)]. We obtain for the three components, $p_{\mathrm{I}}, p_{\mathrm{II}}$, and $p_{\mathrm{III}}$ the following ${ }^{38}$ :

$$
\begin{aligned}
& p_{\mathrm{I}}(t)=\frac{1}{2}\left[\chi_{1}\left(\omega_{1}\right)(1 \mp \cos \theta)+\chi_{2}\left(\omega_{1}\right)(1 \pm \cos \theta)\right] \mathscr{E}_{1} \cos \Phi_{1}+\frac{1}{2}\left[\chi_{1}\left(\omega_{2}\right)(1 \mp \cos \theta)+\chi_{2}\left(\omega_{2}\right)(1 \pm \cos \theta)\right] \mathcal{E}_{2} \cos \Phi_{2}, \\
& p_{\text {II }}(t)=\frac{1}{4} \operatorname{Re} \chi^{(3)}\left(\mathcal{E}_{1} e^{-i \Phi_{2}}+\mathscr{E}_{2} e^{-i \Phi_{1}}\right)\left(1+\frac{i}{\gamma_{3}} \frac{\partial}{\partial t}\right) \mathscr{E}_{1} \mathscr{E}_{2}\left|\frac{\delta}{\gamma}\right|,
\end{aligned}
$$

and

$$
p_{\text {III }}(t)=\frac{1}{4} \operatorname{Re}\left[\chi^{(3)}\left(2 \omega_{1}+\omega_{2}\right) \mathscr{E}_{1} e^{-i\left(2 \Phi_{1}+\Phi_{2}\right)}+\chi^{(3)}\left(\omega_{1}+2 \omega_{2}\right) \mathscr{E}_{2} e^{-i\left(\Phi_{1}+2 \Phi_{2}\right)}\right]\left(1+\frac{i}{\gamma_{3}} \frac{\partial}{\partial t}\right) \mathscr{E}_{1} \mathcal{E}_{2}\left|\frac{\delta}{\gamma}\right|,
$$

where

$$
\begin{aligned}
& \delta=\Omega_{12}-\left(\omega_{1}+\omega_{2}\right), \\
& \chi^{(3)}=\frac{1}{\hbar^{3}} \sum_{m, n} \frac{p_{2 n} p_{n 1} p_{1 m} p_{m 2}}{\delta}\left(\frac{1}{\Omega_{m 2}-\omega_{1}}+\frac{1}{\Omega_{m 2}-\omega_{2}}\right)\left(\frac{1}{\Omega_{n 2}-\omega_{1}}+\frac{1}{\Omega_{n 2}-\omega_{2}}\right), \\
& \chi^{(3)}\left(2 \omega_{1}+\omega_{2}\right)=\frac{1}{\hbar^{3}} \sum_{m, n} \frac{p_{2 n} p_{n 1} p_{1 m} p_{m 2}}{\delta}\left(\frac{1}{\Omega_{m 2}-\omega_{1}}+\frac{1}{\Omega_{m 2}-\omega_{2}}\right)\left(\frac{1}{\Omega_{n 2}+\omega_{1}}+\frac{1}{\Omega_{n 2}-\left(2 \omega_{1}+\omega_{2}\right)}\right),
\end{aligned}
$$

and

$$
\chi^{(3)}\left(\omega_{1}+2 \omega_{2}\right)=\frac{1}{\hbar^{3}} \sum_{m, n} \frac{p_{n 2} p_{n 1} p_{1 m} p_{m 2}}{\delta}\left(\frac{1}{\Omega_{m 2}-\omega_{1}}+\frac{1}{\Omega_{m 2}-\omega_{2}}\right)\left(\frac{1}{\Omega_{n 2}+\omega_{2}}+\frac{1}{\Omega_{n 2}-\left(\omega_{1}+2 \omega_{2}\right)}\right) .
$$

The $\chi$ 's defined above are identical to susceptibilities obtained using perturbation theory, ${ }^{9,16,17}$ and they all have the resonant denominator $\delta$. Consequently, as $\delta \rightarrow 0$ the polarization does not disappear even though the power factor $|\delta / \gamma| \rightarrow 0$.
As mentioned earlier, the components of $p_{I}$ give rise to the dispersion produced by the allowed onephoton transition $|1\rangle-|n\rangle$ and $|2\rangle-|n\rangle$, and are therefore proportional to the populations of states $|1\rangle$ and $|2\rangle$, which are given by $\frac{1}{2}(1 \mp \cos \theta)$ and 
$\frac{1}{2}(1 \pm \cos \theta)$, respectively. The components of $p_{I I}$ also oscillate at the frequency of the driving fields. However, these components can be thought of as the result of a nonlinear mixing of the fields which gives rise to the frequencies $\omega_{1}+\omega_{2}-\omega_{1}$ and $\omega_{1}+\omega_{2}-\omega_{2}$. The out-of-phase terms of $p_{\text {II }}$ are responsible for the two-photon pulse-propagation effects discussed in Sec. IIIC, while the in-phase terms can cause self-focusing or self-defocusing of light beams and self-phase modulation. These effects are usually detrimental to the parametric mixing processes giving frequencies $\omega_{1}+\omega_{2}+\omega_{1}$ and $\omega_{1}+\omega_{2}+\omega_{2}$ and described by $p_{\text {III }}$.

In the limit where $\pi-\theta \ll 1$ and $\mathcal{E}_{1}, \mathcal{E}_{2}, \Phi_{1}$, and $\Phi_{2}$ are constant (which is the usual situation to which time-dependent perturbation theory is applied) Eqs. (65)-(67) reduce to

$$
\begin{aligned}
& p_{\mathrm{I}}(t)=\chi_{2}\left(\omega_{1}\right) \mathcal{E}_{1} \cos \Phi_{1}+\chi_{2}\left(\omega_{2}\right) \mathcal{E}_{2} \cos \Phi_{2}, \\
& p_{\mathrm{II}}(t)=\frac{1}{4} \chi^{(3)}\left(\mathcal{E}_{1}^{2} \mathcal{E}_{2} \cos \Phi_{2}+\mathcal{E}_{1} \mathcal{E}_{2}^{2} \cos \Phi_{1}\right),
\end{aligned}
$$

and

$$
\begin{aligned}
& p_{\text {III }}(t)=\frac{1}{4} \operatorname{Re}[ \chi^{(3)}\left(2 \omega_{1}+\omega_{2}\right) \mathcal{E}_{1}^{2} \mathcal{E}_{2} e^{-i\left(2 \Phi_{1}+\Phi_{2}\right)} \\
&\left.+\chi^{(3)}\left(\omega_{1}+2 \omega_{2}\right) \mathcal{E}_{1} \mathcal{E}_{2}^{2} e^{-i\left(\Phi_{1}+2 \Phi_{2}\right)}\right] .
\end{aligned}
$$

These results, as expected, are identical to those obtained by the usual methods of time-dependent perturbation theory. ${ }^{9,16,17}$

However, the adiabatic following model gives a power-dependent susceptibility as an additional result. Both polarizations $p_{\mathrm{II}}$ and $p_{\mathrm{III}}$ are multiplied by the power factor $|\delta / \gamma|$, where

$\gamma=+\left\{\left[\Omega_{12}-\omega_{1}^{\prime}-\omega_{2}^{\prime}+\left(\Delta E_{1}-\Delta E_{2}\right) / \hbar\right]^{2}+\left(\kappa \mathcal{E}_{1} \mathcal{E}_{2}\right)^{2}\right\}^{1 / 2}$.

It is very satisfying that the power dependence is completely described by this simple power factor, i.e., the power-dependent susceptibilities are given by the usual well-known results multiplied by $|\delta / \gamma|$. The power factor is equal to unity at $\mathcal{E}_{1}, \mathcal{E}_{2} \rightarrow 0$, while at very strong fields, where $\delta$ can be neglected compared to the Stark shifts, the power factor has a saturation type of behavior. The behavior at intermediate field strengths, where $\delta$ is comparable to the Stark shifts, is more complicated, and $|\delta / \gamma|$ may either increase or decrease depending on the sign of the Stark shift $\Delta E_{1}-\Delta E_{2}$ with respect to $\delta$. The optimum field strengths will depend on the parameters for a given problem. We note that a power-dependent susceptibility for third-harmonic generation (treated in Appendix A) has recently been calculated by Chang ${ }^{11}$ using the techniques of high-intensity quantum electrodynamics. Also, Stappaerts $^{39}$ has made a recent study of frequency shifts and saturation phenomena in harmonic generation at high field strengths.

The out-of-phase terms of $p_{\text {II }}$ and $p_{\text {III }}$ are unique to the AF model. These terms describe the energy flow between the propagating electromagnetic waves and the coherent excitation of the two-photon resonance. When the light intensity increases, energy flows from the light waves into the two-photon coherent excitation of the atom, and when the intensity decreases, energy flows from the atomic excitation back into the light wave. This energy transfer is responsible for the nonlinear pulse velocity discussed in Sec. III C. This type of argument has been used to obtain the group velocity for the one-photon resonance propagation problem. ${ }^{6}$ For that case, the group velocity can also be obtained from linear dispersion theory. However, linear dispersion theory cannot be applied to the two-photon problem, and the pulse velocity can only be derived from the out-of-phase components of the polarization.

\section{Pulse propagation}

In this section we will consider the propagation of a circularly polarized, single-frequency light pulse nearly resonant with a two-photon resonance. As shown in Appendix B 2, this choice simplifies the problem considerably in that $p_{\mathrm{III}}=0$ and only one field $\overrightarrow{\mathrm{E}}$ is coupled to Maxwell's equations. The electric field $\vec{E}$ of the pulse is given by

$$
\overrightarrow{\mathrm{E}}=\mathcal{E}(\hat{x} \cos \Phi+\hat{y} \sin \Phi),
$$

with

$$
\Phi=\omega t+\phi-k z .
$$

To a very good approximation, the pulse propagation is described by the usual reduced wave equations for the field amplitude $\mathcal{E}$ and slowly varying phase $\phi,^{8}$

$$
\begin{aligned}
& \frac{\partial \mathcal{E}}{\partial z}+\frac{1}{c} \frac{\partial \mathcal{E}^{\prime}}{\partial t}=-\frac{2 \pi \omega}{c} v, \\
& \frac{\partial \phi}{\partial z}+\frac{1}{c} \frac{\partial \phi}{\partial t}=-\frac{2 \pi \omega}{c} \frac{u}{\mathcal{E}} .
\end{aligned}
$$

In Eqs. (77), $v$ designates the out-of-phase component of the polarization, and $u$ designates the in-phase component.

The components $u$ and $v$ are given by the AF solutions obtained from Eqs. (B18a) and (B18b) of Appendix B as

$$
\begin{aligned}
u= & \frac{1}{2}\left(\chi_{1}+\chi_{2}\right) N \mathcal{E}+\frac{1}{2}\left(\chi_{2}-\chi_{1}\right) N \mathcal{E}\left( \pm \gamma_{3} / \gamma\right) \\
& +\frac{1}{2} \chi^{(3)} N \mathcal{E}^{3}|\delta / \gamma|
\end{aligned}
$$

and 


$$
v=\frac{1}{2} \chi^{(3)} \frac{N|\delta| \mathcal{E}}{\gamma_{3}} \frac{\partial}{\partial t} \frac{\mathcal{E}^{2}}{\gamma},
$$

where $N$ is the atomic number density. By taking the partial derivative with respect to time of Eq. (77b) and defining the instantaneous frequency $\omega^{\prime}$ as

$$
\omega^{\prime} \equiv \omega+\partial \phi / \partial t
$$

Eq. (77b) can be changed to

$$
\frac{\partial \omega^{\prime}}{\partial z}+\frac{1}{c} \frac{\partial \omega^{\prime}}{\partial t}=-\frac{2 \pi \omega}{c} \frac{\partial}{\partial t} \frac{u}{\mathcal{E}}
$$

Then, substituting Eqs. (78) for $u$ and $v$ into Eqs. (77a) and (80), we obtain

$$
\begin{aligned}
\frac{\partial \mathcal{E}}{\partial z}+\frac{1}{c} \frac{\partial \mathcal{E}}{\partial t}= & -\frac{2 \pi \omega N \chi^{(3)}|\delta|}{c \gamma^{3}} \\
& \times\left(\left(\Omega_{12}-2 \omega^{\prime}\right) \mathcal{E}^{2} \frac{\partial \mathcal{E}}{\partial t}+\mathcal{E}^{3} \frac{\partial \omega^{\prime}}{\partial t}\right), \\
\frac{\partial \omega^{\prime}}{\partial z}+\frac{1}{c} \frac{\partial \omega^{\prime}}{\partial t}= & -\frac{2 \pi \omega N \chi^{(3)}|\delta|}{c \gamma^{3}} \\
& \times\left(\left(\Omega_{12}-2 \omega^{\prime}\right) \mathcal{E}^{2} \frac{\partial \mathcal{E}}{\partial t}+\mathcal{E}^{3} \frac{\partial \omega^{\prime}}{\partial t}\right) \frac{\Omega_{12}-2 \omega^{\prime}}{\mathscr{E}} .
\end{aligned}
$$

When Eqs. (81) are rewritten in terms of the distance $\zeta$ and the reduced time $\tau$, where

$$
\begin{aligned}
& \zeta=z, \\
& \tau=t-z / c,
\end{aligned}
$$

they show the important relationship

$$
\frac{\partial \omega^{\prime}}{\partial \zeta}=\frac{\Omega_{12}-2 \omega^{\prime}}{\mathcal{E}} \frac{\partial \mathcal{E}}{\partial \zeta},
$$

which can be integrated as

$$
-\left.\frac{1}{2} \ln \left(\Omega_{12}-2 \omega^{\prime}\right)\right|_{\zeta=0} ^{\zeta=z}=\left.\ln \mathcal{E}\right|_{\substack{\zeta=z \\ \zeta=0}} ^{\substack{\zeta \\=0 .}}
$$

Evaluating Eq. (84), we obtain

$$
\frac{\left[\Omega_{12}-2 \omega^{\prime}(z, \tau)\right]}{\left[\Omega_{12}-2 \omega^{\prime}(0, \tau)\right]}=\left(\frac{\mathcal{E}(0, \tau)}{\mathcal{E}(z, \tau)}\right)^{2} .
$$

This is a general relationship, independent of intensity, frequency-offset, propagation distance, and the strength of the interaction $N \chi^{(3)}$. It states that under the AF approximation for two-photon resonance, pulse-reshaping effects and self-phasemodulation effects are always strongly coupled and cannot be treated separately.

It is instructive to demonstrate, however, that Eq. (85) reduces to the simple result for selfphase modulation, ${ }^{8}$ in the limit of very little pulse reshaping. Equation (85) can be expanded as

$$
\frac{\partial \phi}{\partial t}(z, \tau)=\left.\frac{\delta z}{\mathscr{E}(0, \tau)} \frac{\partial \mathcal{E}(\zeta, \tau)}{\partial \zeta}\right|_{\zeta=0},
$$

and from Eq. (81a),

$$
\left.\frac{\partial \mathcal{E}(\zeta, \tau)}{\partial \zeta}\right|_{\zeta=0}=-\frac{2 \pi \omega N \chi^{(3)}|\delta| \delta \mathcal{E}^{2}(0, \tau)}{c \gamma^{3}} \frac{\partial \mathcal{E}(0, \tau)}{\partial \tau}
$$

Thus,

$$
\frac{\partial \phi}{\partial \tau}=-\frac{\omega}{c} z \frac{\pi N \chi^{(3)}|\delta| \delta^{2}}{\gamma^{3}} \frac{\partial \mathcal{E}^{2}(0, \tau)}{\partial \tau} .
$$

The above can also be obtained directly using Eq. (81b), and noticing that $\left(\partial \omega^{\prime} / \partial \tau\right)=0$ at $z=0$. Equation (88) can now be written in the familiar form

$$
\frac{\partial \phi}{\partial \tau}=-\frac{1}{2} \frac{\omega}{c} z \epsilon_{2}\left|\frac{\delta}{\gamma}\right|^{3} \frac{\partial \mathcal{E}^{2}(0, \tau)}{\partial \tau},
$$

where the nonlinear dielectric constant $\epsilon_{2}$ is defined in the usual way (low-intensity limit)

$$
\epsilon=\epsilon_{0}+\epsilon_{2} \mathcal{E}^{2},
$$

and is given by ${ }^{40}$

$$
\epsilon_{2}=2 \pi N \chi^{(3)} \text {. }
$$

In the low-intensity limit, $|\delta / \gamma| \rightarrow 1$, and Eq. (89) is identical to the conventional result. ${ }^{8}$ It is interesting to observe that, while the power factor for the polarization in Eqs. (66) and (67) is $|\delta / \gamma|$, the intensity-dependent factor in Eq. (89) is $|\delta / \gamma|^{3}$, even though in the low-intensity limit the same quantity $\chi^{(3)}$ is involved in both cases. It is also clear, from the derivation above, that even with the proper power-dependent factor, Eq. (89) is still an approximation of limited validity, and, in general, Eq. (85) should be used. The situation here is quite similar to the near-resonance onephoton propagation problem. ${ }^{\text {? }}$

The nonlinear pulse velocity $v_{p}$ implicit in Eq. (81a) can be displayed by rewriting the equation as

$$
\frac{\partial \mathcal{E}}{\partial z}+\frac{1}{v_{p}} \frac{\partial \mathcal{E}}{\partial t}=-\frac{2 \pi \omega N \chi^{(3)}|\delta| \mathcal{E}^{3}}{c \gamma^{3}} \frac{\partial \omega^{\prime}}{\partial t}
$$

where

$$
\frac{1}{v_{p}}=\frac{1}{c}\left(1+\frac{2 \pi \omega N \chi^{(3)}|\delta|\left(\Omega_{12}-2 \omega^{\prime}\right)}{\gamma^{3}} \mathcal{E}^{2}\right) .
$$

If it were possible to neglect self-phase modulation by setting the right-hand side of Eq. (91) equal to zero, the pulse would travel as a simple wave with the nonlinear pulse velocity $v_{p}$. It is instructive to compare Eq. (92) with the one-photon nonlinear pulse velocity. ${ }^{7,8}$ In the low-intensity limit, the one-photon nonlinear velocity becomes the group velocity $v_{g}$, in agreement with linear dispersion theory. The pulse in that case propagates as a simple wave, with pulse reshaping due to the extreme frequency dispersion of $v_{g}$. In the two- 
photon resonance problem, no such linear pulsereshaping effects are present since $v_{p} \rightarrow c$ in the low-intensity limit. All pulse-reshaping effects then are nonlinear in nature and must be studied using the coupled Eqs. (81).

In order to demonstrate the pulse reshaping implied by the coupled Eqs. (81) we numerically integrate these equations for the specific example of pulse propagation in $\mathrm{Rb}$ vapor. ${ }^{41}$ The two-photon resonance considered is from the $5 S_{1 / 2}$ ground state to the $5 D_{5 / 2}$ excited state, as illustrated in Fig. 4. Also, because of the near coincidence with the single-photon transition from the $5 S_{1 / 2}$ to the $5 P_{3 / 2}$ state, only the $5 P_{3 / 2}$ state need be considered as an intermediate state. Thus, for illustrative purposes we can consider the $\mathrm{Rb}$ atom as the three-level system shown in Fig. 4. For this example, $\Omega_{12} / 2 \pi c=25703.5 \mathrm{~cm}^{-1} ; \delta / 2 \pi c=2 \mathrm{~cm}^{-1}$; $\Delta / 2 \pi c=34.2 \mathrm{~cm}^{-1} ; p_{2 n}=7.5 \times 10^{-18}$ esu $\left[\sigma^{-}\right.$transi tion, $\left.5 S_{1 / 2}\left(m=-\frac{1}{2}\right) \multimap 5 P_{3 / 2}\left(m=-\frac{3}{2}\right)\right] ;^{42} p_{n 1}=2.0$ $\times 10^{-18}$ esu $\left[\sigma^{-}\right.$transition, $5 P_{3 / 2}\left(m=-\frac{3}{2}\right)-5 D_{5 / 2}$ $\left.\left(m=-\frac{5}{2}\right)\right] .{ }^{42}$ The atomic number density $N$ initially in the $5 S_{1 / 2}\left(m=-\frac{1}{2}\right)$ ground state is one-half the total number density, and in the calculation $N$ is set equal to $2 \times 10^{14}$ atoms $/ \mathrm{cm}^{3}$, corresponding to the readily obtainable $\mathrm{Rb}$ cell temperature of $180^{\circ} \mathrm{C}$.

Figure 5(a) shows the predicted reshaping of a Gaussian pulse arising from passage through a $60-\mathrm{cm} \mathrm{Rb}$ cell with the above parameters. The input peak intensity is $8 \mathrm{MW} / \mathrm{cm}^{2}$, and the input pulse width (full width at half-maximum) is 200 psec.

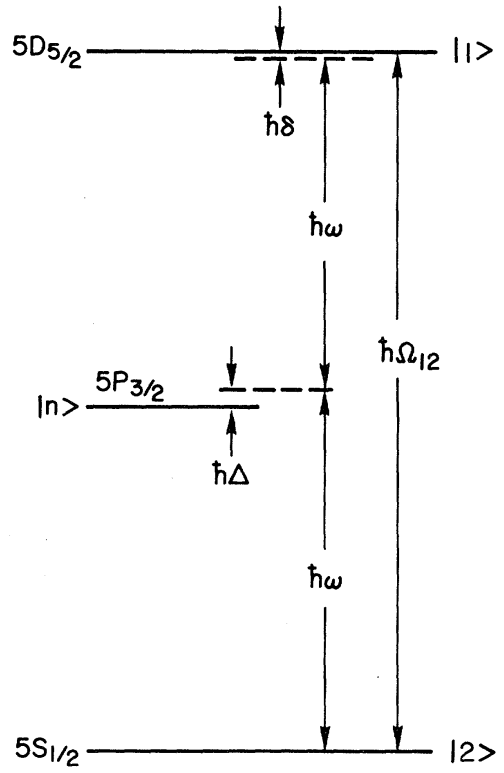

FIG. 4. Energy levels of Rb relevant to the two-photon transition $\Omega_{12} . \delta / 2 \pi c=2 \mathrm{~cm}^{-1}$, and $\Delta / 2 \pi c=34.2 \mathrm{~cm}^{-1}$.
The output pulse is of lower peak intensity, and a discontinuity (optical shock) ${ }^{7}$ is forming on the trailing edge. Any further propagation sharpens the trailing edge so severely that the AF conditions are violated and Eqs. (81) no longer apply. If $\delta<0$, instead of $\delta>0$ as in this example, the discontinuity forms on the leading edge of the pulse. In contrast, for pulse reshaping caused by a single-photon resonance, the peak intensity is always increased by pulse-compression effects, and the discontinuity always appears on the leading edge of the pulse. Similar to the single-photon case, for the two-photon example there are two effects responsible for the pulse reshaping: (a) the intensitydependent pulse velocity, and (b) the self-phase modulation. For the two-photon resonance, the effect of self-phase modulation is dominant, even though the low-intensity group velocity has no frequency dispersion.

The calculated self-phase modulation caused by
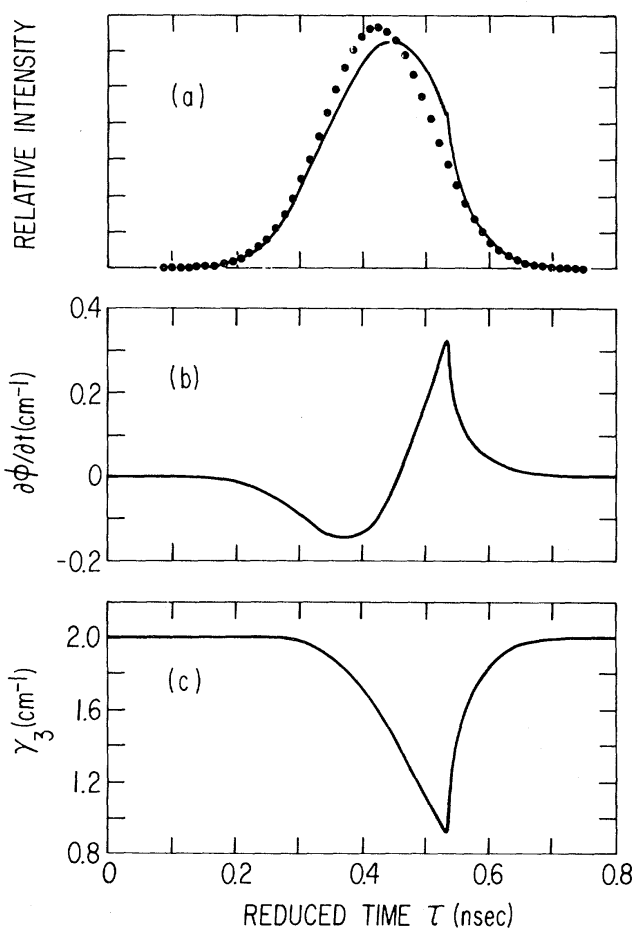

FIG. 5. Calculated two-photon pulse reshaping and self-phase modulation. (a) Input pulse (dotted line) with peak intensity of $8 \mathrm{MW} / \mathrm{cm}^{2}$ and $0.2-\mathrm{nsec}$ pulse width (FWHM). Calculated output pulse (solid line) after passage through $60-\mathrm{cm} \mathrm{Rb}$ cell with a total atomic number density of $4 \times 10^{14} \mathrm{~cm}^{-3} . \quad \delta / 2 \pi c=2 \mathrm{~cm}^{-1}$, and $\Delta / 2 \pi c$ $=34.2 \mathrm{~cm}^{-1}$, as shown in Fig. 4 ; (b) Calculated selfphase modulation $(\partial \phi / \partial t)$ caused by passage through the $\mathrm{Rb}$ cell; (c) Calculated offset frequency $\gamma_{3}$ at the end of the $\mathrm{Rb}$. cell. $\gamma_{3}$ includes both the self-phase modulation of the propagating wave and the optical Stark shift of the two-photon resonance. 
the passage through the $\mathrm{Rb}$ vapor is shown in Fig. $5(b)$. This result is, of course, in exact agreement with that predicted by Eq. (85) together with Fig. 5(a). The instantaneous frequency is changed dramatically by the passage, and the bandwidth has increased tenfold. Consequently, these pulses could be compressed to $\sim 20$ psec by passage through a dispersive delay line. ${ }^{43}$

Figure 5(c) shows the time dependence of the instantaneous frequency offset $\gamma_{3}$ at the end $(z=60$ $\mathrm{cm}$ ) of the $\mathrm{Rb}$ cell. The offset is changed by both the self-phase modulation and the optical Stark shift as explained by Eq. (B15c). The Stark shift is proportional to the light intensity, and for this example an intensity of $8 \mathrm{MW} / \mathrm{cm}^{2}$ shifts the twophoton resonance to lower frequencies by 0.65 $\mathrm{cm}^{-1}$. The Stark shift is a local effect, but the self-phase modulation is a cumulative effect. Thus, at the entrance $(z=0)$ of the cell, the time dependence of $\gamma_{3}$ is entirely due to the Stark shift; at the end of the cell, the Stark shift and the selfphase modulation are comparable in their contribution to $\gamma_{3}$. The Stark shift is proportional to the pulse intensity, and the self-phase modulation is proportional to the derivative of the pulse intensity. Thus for this example, at the leading edge of the pulse the two effects have opposite signs, while at the trailing edge they have the same sign. This accounts for the resultant shape of $\gamma_{3}$ at the end of the cell $(z=60 \mathrm{~cm})$, where the instantaneous frequency is swept closer to the line for the entire pulse duration. ${ }^{44}$

This example also demonstrates the importance of the factor $|\delta / \gamma|^{3}$ previously discussed in Eq. (89), which is valid when pulse reshaping is small as at the leading edge of the pulse [Fig. 5(a)]. The calculated result using the conventional self-phasemodulation formula (without the factor $|\delta / \gamma|^{3}$ ) differs from that in Fig. 5(b) in at least two aspects. First, the maximum frequency shift is significantly greater than that predicted by the simple formula. Second, the maximum frequency shift occurs later in time than the maximum derivative of the pulse intensity; the simple formula predicts that the two occur simultaneously. It is easy to see that inclusion of the factor $|\delta / \gamma|^{3}$ removes both deficiencies. The parameter $\gamma$, which for small $z$ is mainly determined by the optical Stark shift, is smaller than $\delta$. Thus, the power factor $|\delta / \gamma|$ is larger than 1 and increases with time at the leading edge of the pulse. As a result, the maximum frequency shift is higher and occurs later. With the factor $|\delta / \gamma|^{3}$, the prediction of Eq. (89) is in fair agreement with Fig. $5(\mathrm{~b})$ in the region where pulse reshaping can be ignored.

As discussed earlier, when the intermediate state is below the two-photon excited state, a large gain for lasing from the excited state to the intermediate state can occur in coherent two-photon propagation problems. For our case, the problem of lasing to the intermediate state at first appears to be severe. The number density $N_{1}$ of atoms in the two-photon excited state is given for the AF model (with $\gamma_{3}>0$ ) by the relationship

$$
N_{1}=\frac{1}{2} N(1-\cos \theta) \text {. }
$$

Evaluating Eq。(93) at $z=0$ with the above parameters, we obtain $N_{1}=3.7 \times 10^{12}$ atoms $/ \mathrm{cm}^{3}$. This number density gives the very large gain coefficient $g=10.5 \mathrm{~cm}^{-1}$ for lasing to the $5 P_{3 / 2}$ intermediate state. Thus, for a $60-\mathrm{cm}$ cell, the total gain would at first sight seem to be the enormous value of $e^{630}$. However, there are two important factors which reduce this large gain and appear to prevent lasing. The first is the large Stark shift of the transition frequency $\left[\Omega_{1 n}-\left(\Delta E_{1}-\Delta E_{n}\right) / \hbar\right]$. This causes the transition frequency to differ by $0.8 \mathrm{~cm}^{-1}$ over the pulse envelope. The second factor is that, under very high gain conditions, light travels slowly. ${ }^{45}$ Consequently, a gain of $g$ $=10.5 \mathrm{~cm}^{-1}$ would imply a group velocity of $\frac{1}{75} c$, and the emitted light would fall out of step with the propagating pulse which is traveling at essentially $c$. As the pulse is only $200 \mathrm{psec}$ long $(6 \mathrm{~cm}$ in length), the emitted light will get out of resonance with the Stark broadened line in less than $0.01 \mathrm{~cm}$. Thus, the total gain is reduced to negligible amounts and lasing does not seem probable.

The situation with respect to lasing is not as favorable for the earlier two-photon self-inducedtransparency experiment. ${ }^{18}$ There, at the peak of the pulse all the resonant atoms were in the two-photon excited state, giving a gain coefficient for lasing to the intermediate $P_{3 / 2}$ state of $22 \mathrm{~cm}^{-1}$. As the cell was $140 \mathrm{~cm}$ long, this would imply a total gain of the enormous number $e^{3080}$. Here, the Stark shift is negligible. Again, the emitted light would travel extremely slowly owing to the high gain, with a group velocity of only $\frac{1}{100} c$. For this experiment the pulse width was 15 nsec, and the emitted light could travel $4.5 \mathrm{~cm}$ during the pulse duration. This situation reduces the gain to $g \sim e^{100}$, and lasing to the $4 P_{3 / 2}$ state appears to be a serious problem.

The discussions above are, of course, highly simplified. Our aim is merely to point out the complications inherently connected with two-photon propagation problems. For example, in our discussion on group velocity mismatch, we have assumed perfect adiabatic following with no population left in the excited state after the input pulse has gone by. In fact there will always be some residual excitation, because of the approximate nature of the AF solution. However, as the sum 
frequency is tuned farther away from the two-photon resonance, the AF conditions are better satisfied and the residual excitation is decreased. In practice, for off-resonance propagation, the amount of residual excitation is mainly determined by the relaxation processes. Also, while a slow pulse propagation velocity of $\frac{1}{15} c$ has been experimentally observed, ${ }^{6}$ the much slower velocities above are calculated and remain to be confirmed experimentally. All these and probably other factors need further experimental and theoretical study. Meanwhile, two-photon propagation experiments must be interpreted cautiously.

\section{SUMMARY}

The application of a vector model to the two-photon resonance in the two-level approximation has been demonstrated and explained in Sec. II. The atomic polarization has been calculated in terms of the components of the $\overrightarrow{\mathbf{r}}$ vector and shown to have three contributions, $p_{I}, p_{1 I}$, and $p_{1 I I}$, each having a different physical interpretation.

The $p_{\mathrm{I}}$ components oscillate in phase with the driving fields. These components are due to the allowed one-photon transitions from the two-photon ground state to the intermediate states and from the two-photon excited state to the intermediate states. Consequently, the polarization arising from these terms is proportional to the atomic populations in the ground and excited states. As shown in the example of Sec. III, these terms can cause strong self-phase modulation.

The $p_{\text {II }}$ components are those traditionally considered for two-photon coherent-pulse propagation. For $p_{\text {II }}$, the out-of-phase term is proportional to $r_{2}$ and is responsible for the energy exchange between the atoms and the propagating waves; it is responsible for slow pulse velocities and pulse reshaping. The in-phase component is proportional to $r_{1}$ and is analogous to its counterpart in onephoton processes. This component can cause important effects such as self-focusing, self-defocusing, and self-phase modulation. As demonstrated in Sec. III, this in-phase term is mainly responsible for the large self-phase modulation which accompanies two-photon pulse propagation.

The $p_{\text {III }}$ components cause the two-photon resonantly enhanced generation of mixed frequencies. For two-photon propagation problems with linearly polarized light beams, with frequencies far from any resonances to the intermediate states, $p_{\mathrm{III}}$ can be as large as $p_{\mathrm{I}}$ and $p_{\mathrm{II}}$. Consequently, $p_{\mathrm{III}}$ must be considered for propagation problems of this type. However, as shown in Appendix B, for circularly polarized light $p_{\mathrm{III}}=0$, and propagation problems are dramatically simplified. If linearly polarized light must be used, it is possible to make $p_{\text {III }}$ negligible compared to $p_{\text {II }}$ by using resonant enhancement, where an input frequency is nearly resonant with a transition from the twophoton ground state to the intermediate state. This is a viable procedure for the study of local effects (photon echoes, optical nutation, adiabatic rapid passage, and optical Stark shifts). In this case, however, the study of two-photon pulse-propagation problems may be complicated by the problem of lasing from the two-photon excited state to the intermediate state.

In Sec. III the adiabatic following (AF) approximation is applied to the vector model for the twophoton resonance. The AF approximation provides simple expressions for the components of the $\overrightarrow{\mathbf{r}}$ vector of the two-photon, two-level atom. Using these AF results for $\overrightarrow{\mathrm{r}}$, the atomic polarization in terms of $p_{I}, p_{\text {II }}$, and $p_{\text {III }}$ is thereby obtained.

In the limit where the angle between $\vec{r}$ and the $3^{\prime \prime}$ axis is time independent and very small (lowintensity limit), the polarization components are simplified. The $p_{\text {I }}$ terms reduce to the usual result for the polarization from the two-photon ground state to the intermediate states. The $p_{\text {II }}$ terms reduce to the usual Kerr-type nonlinear polarization, $, 9,17,40$ and the $p_{\text {III }}$ terms give the nonlinear mixing that one calculates from time-dependent perturbation theory. ${ }^{9,16,17}$ Thus, in terms of the vector model, the condition described by time-dependent perturbation theory corresponds to $\overrightarrow{\mathbf{r}}$ adiabatically following $\vec{\gamma}$ in the small-angle limit.

The above situation allows for a very natural extension of the AF results to large angles as well. This case corresponds to the high-intensity situation very close to the two-photon resonance. Previously, this situation has been poorly understood, because here the ordinary perturbation approach is invalid. The AF solution gives a very simple result for these conditions; the power-dependent nonlinear susceptibilities are equal to the usual power-independent susceptibilities multiplied by the power factor $|\delta / \gamma|$. Thus, one can now calculate precisely the nonlinear mixing obtained for resonantly enhanced conditions with high input intensities.

The AF results also give out-of-phase components of polarization owing to the time dependence of the input intensities or frequencies. These components control the energy exchange between the atoms and the propagating waves and are responsible for the nonlinear pulse velocity derived in Sec. III C. There it is shown that, similar to one-photon resonances, two-photon near-resonant propagation can in general be described by the coupled amplitude and phase equations. An analytic rela- 
tionship between the pulse reshaping and the selfphase modulation is derived showing the strong coupling between the two effects. For short propagation distances where pulse reshaping is negligible, the self-phase modulation is shown to be equal to the conventional low-intensity result multiplied by the factor $|\delta / \gamma|^{3}$. Since the power factor $|\delta / \gamma|$ can be larger than unity, the resultant phase modulation can be substantially larger than that calculated from the low-intensity formula.

To illustrate this two-photon propagation process, the coupled phase and amplitude equations are solved numerically for a realistic case. Strong pulse reshaping and self-phase modulation are indeed obtained as expected, and the results are discussed.

Finally, we discuss the often-neglected question of lasing from the two-photon excited state to the lower-energy intermediate state. It is shown that this can be a serious problem in two-photon pulsepropagation experiments. However, there are two effects which together seem to dramatically reduce the effective gain and in some cases eliminate the problem. These coupled effects of the optical Stark shift, together with the group velocity mismatch, need further study before conclusive statements on this question can be made.

We would like to acknowledge J. A. Armstrong's suggestion to extend the theory to circularly polarized light, and J. J. Wynne's careful reading of the manuscript and his numerous helpful comments.

\section{APPENDIX A: SINGLE-FREQUENCY CASE FOR LINEARLY POLARIZED LIGHT}

Here we treat the case where the second harmonic of the input field is near resonant to $\Omega_{12}$. Let the input field be

$$
\overrightarrow{\mathrm{E}}=\hat{x} \mathcal{E} \cos \Phi,
$$

with

$$
\Phi=\omega t+\phi-k z
$$

and

$$
2 \omega \simeq \Omega_{12} .
$$

Most of the expressions in Sec. II apply if we make the following changes: $\mathcal{E}_{1} \rightarrow \mathcal{E}, \mathcal{E}_{2} \rightarrow 0, \omega_{2} \rightarrow \omega$, and $\omega_{1} \rightarrow \omega$. Some, however, do not follow this rule; see, for example, the expression for $\kappa$. For completeness we will display the pertinent equations:

$$
\begin{aligned}
& \gamma_{1}=-\kappa \mathcal{E}^{2}, \\
& \gamma_{2}=0, \\
& \gamma_{3}=\Omega_{12}-2 \omega^{\prime}+\left(\Delta E_{1}-\Delta E_{2}\right) / \hbar,
\end{aligned}
$$

with

$$
\omega^{\prime}=\omega+\partial \phi / \partial t
$$

and

$$
\kappa=\frac{1}{2 \hbar^{2}}\left|\sum_{n} \frac{p_{1 n} p_{n 2}}{\Omega_{n 2}-\omega}\right| .
$$

The expressions for the polarization are

$$
\begin{gathered}
p_{\mathrm{I}}(t)=\frac{1}{2}\left[\chi_{1}\left(1+r_{3}\right)+\chi_{2}\left(1-r_{3}\right)\right] \mathscr{E} \cos \Phi, \\
p_{\mathrm{II}}(t)=\operatorname{Re} 2 \hbar \kappa\left(r_{1}-i r_{2}\right) \mathcal{E} e^{-i \Phi}, \\
p_{\mathrm{III}}(t)=\operatorname{Re}\left(r_{1}-i r_{2}\right) \sum_{n} \frac{p_{2 n} p_{n 1}}{2 \hbar}\left(\frac{1}{\Omega_{2 n}+\omega}+\frac{1}{\Omega_{n 2}-3 \omega}\right) \\
\times \mathscr{E} e^{-i(3 \Phi+\beta)} .
\end{gathered}
$$

Using the AF solutions for the components of $\vec{r}$, we can rewrite Eq. (A4) as $^{38}$

$$
\begin{aligned}
& p_{\mathrm{I}}(t)=\frac{1}{2}\left[\chi_{1}(1 \mp \cos \theta)+\chi_{2}(1 \pm \cos \theta)\right] \mathcal{E} \cos \Phi, \\
& p_{\mathrm{II}}(t)=\frac{1}{4} \operatorname{Re} \chi^{(3)}(\omega) \mathcal{E} e^{-i \Phi}\left(1+\frac{i}{\gamma_{3}} \frac{\partial}{\partial t}\right) \mathcal{E}^{2}\left|\frac{\delta}{\gamma}\right|, \\
& p_{\mathrm{III}}(t)=\frac{1}{4} \operatorname{Re} \chi^{(3)}(3 \omega) \mathcal{E} e^{-i 3 \Phi}\left(1+\frac{i}{\gamma_{3}} \frac{\partial}{\partial t}\right) \mathcal{E}^{2}\left|\frac{\delta}{\gamma}\right|,
\end{aligned}
$$

where

$$
\begin{aligned}
& \chi_{i}=\sum_{n} \frac{p_{i n} p_{n i} 2 \Omega_{n i}}{\hbar\left(\Omega_{n i}^{2}-\omega^{2}\right)}, \\
& \chi^{(3)}(\omega)=\frac{2}{\hbar^{3}} \sum_{m, n} \frac{p_{2 n} p_{n 1} p_{1 m} p_{m 2}}{\delta\left(\Omega_{m 2}-\omega\right)\left(\Omega_{n 2}-\omega\right)},
\end{aligned}
$$

and

$\chi^{(3)}(3 \omega)=\frac{1}{\hbar^{3}} \sum_{m, n} \frac{p_{2 n} p_{n 1} p_{1 m} p_{m 2}}{\delta\left(\Omega_{m 2}-\omega\right)}\left(\frac{1}{\Omega_{n 2}+\omega}+\frac{1}{\Omega_{n 2}-3 \omega}\right)$.

For small angles, i.e., $\pi-\theta \ll 1$, and $\mathcal{E}$ and $\Phi$ constant, the expression for $p(t)$ can be reduced to

$$
\begin{aligned}
& p_{\mathrm{I}}(t)=\chi_{2} \mathcal{E} \cos \Phi, \\
& p_{\mathrm{II}}(t)=\frac{1}{4} \chi^{(3)}(\omega) \mathcal{E}^{3} \cos \Phi, \\
& p_{\mathrm{III}}(t)=\frac{1}{4} \operatorname{Re} \chi^{(3)}(3 \omega) \mathcal{E}^{3} e^{-i 3 \Phi},
\end{aligned}
$$

which is identical to the results obtained using perturbation theor $y^{9,17}$ and diagrammatic techniques. ${ }^{16}$

Note that the power factor $|\delta / \gamma|$, at exact resonance $\left(\gamma_{3}=0\right)$, reduces to $\left|\delta /\left(\kappa \delta^{2}\right)\right|$. Thus, at exact resonance both $p_{\text {II }}$ and $p_{\text {III }}$ have a linear dependence on $\mathcal{E}$, in contrast to the $\mathcal{E}^{3}$ dependence obtained from perturbation theory. Therefore, our on-resonance results have the same power dependence as that predicted by Chang. ${ }^{11}$ 


\section{APPENDIX B: CIRCULARLY POLARIZED LIGHT}

\section{Two-frequency case}

Here we treat the case of circularly polarized light under the conditions where the mixing term $p_{\text {III }}$ will be absent. Let the input field be given by

$$
\overrightarrow{\mathrm{E}}=\overrightarrow{\mathrm{E}}_{1}+\overrightarrow{\mathrm{E}}_{2} \text {, }
$$

with

$$
\overrightarrow{\mathrm{E}}_{1}=\mathcal{E}_{1}\left(\hat{x} \cos \Phi_{1}+\hat{y} \sin \Phi_{1}\right)
$$

and

$$
\overrightarrow{\mathrm{E}}_{2}=\mathcal{E}_{2}\left(\hat{x} \cos \Phi_{2}+\hat{y} \sin \Phi_{2}\right) .
$$

Because we are considering only $\sigma$ transitions under the conditions that no frequency mixing occurs, the matrix elements of the electric dipole moment between the intermediate states $|n\rangle$ and the states $|1\rangle$ and $|2\rangle$ are given by

$$
\overrightarrow{\mathrm{p}}_{n 1}=p_{n 1}(\hat{x}+i \hat{y}) / \sqrt{2}
$$

and

$$
\overrightarrow{\mathrm{p}}_{2 n}=p_{2 n}(\hat{x}+i \hat{y}) / \sqrt{2} \text {. }
$$

The relevant matrix elements of the effective Hamiltonian in the primed reference frame are given by

$$
\begin{aligned}
& \mathcal{C}_{e_{21}}^{\prime}=\mathcal{H}_{e_{12}}^{*}, \\
& \mathcal{H}_{e_{11}}^{\prime}=\hbar \Omega_{1}+\Delta E_{1}, \\
& \mathcal{H}_{e_{22}}^{\prime}=\hbar \Omega_{2}+\Delta E_{2} .
\end{aligned}
$$

The optical Stark shifts of states $|1\rangle^{\prime}$ and $|2\rangle^{\prime}$ are given by

$$
\Delta E_{1}=-\frac{1}{2}\left[\chi_{1}\left(\omega_{1}\right) \mathcal{E}_{1}^{2}+\chi_{1}\left(\omega_{2}\right) \mathcal{E}_{2}^{2}\right]
$$

and

$$
\Delta E_{2}=-\frac{1}{2}\left[\chi_{2}\left(\omega_{1}\right) \mathcal{E}_{1}^{2}+\chi_{2}\left(\omega_{2}\right) \mathcal{E}_{2}^{2}\right] .
$$

The symbols $\chi_{1}\left(\omega_{1}\right)$ and $\chi_{2}\left(\omega_{1}\right)$ represent the linear electric susceptibility for circularly polarized light of angular frequency $\omega_{1}$ of a single atom in state $|1\rangle$ or $|2\rangle$, respectively:

$$
\begin{aligned}
& \chi_{1}\left(\omega_{1}\right)=\sum_{n} \frac{p_{n n} p_{n 1}}{\hbar\left(\Omega_{n 1}+\omega_{1}\right)}, \\
& \chi_{2}\left(\omega_{1}\right)=\sum_{n} \frac{p_{2 n} p_{n 2}}{\hbar\left(\Omega_{n 2}-\omega_{1}\right)} .
\end{aligned}
$$

For circularly polarized light the off-diagonal matrix element $\mathcal{H}_{e_{12}}^{\prime}$ has terms oscillating at the frequencies $2 \omega_{1}, 2 \omega_{2}$, and $\omega_{1}+\omega_{2}$ only; thus, for circularly polarized light, the counter-rotating-wave approximation is not required. The off-diagonal matrix element is given completely by

$$
\begin{aligned}
\mathcal{H}_{e_{12}}^{\prime}=-\sum_{n}\left\{\frac{p_{1 n} p_{n 2}}{4 \hbar}\left(\Omega_{n 1}+\Omega_{n 2}\right)[\right. & \frac{\mathcal{E}_{1}^{2} e^{-i 2 \Phi_{1}}}{\left(\Omega_{n 1}+\omega_{1}\right)\left(\Omega_{n 2}-\omega_{1}\right)}+\frac{\mathcal{E}_{2}^{2} e^{-i 2 \Phi_{2}}}{\left(\Omega_{n 1}+\omega_{2}\right)\left(\Omega_{n 2}-\omega_{2}\right)} \\
& \left.\left.+\mathcal{E}_{1} \mathcal{E}_{2} e^{-i\left(\Phi_{1}+\Phi_{2}\right)}\left(\frac{1}{\left(\Omega_{n 1}+\omega_{1}\right)\left(\Omega_{n 2}-\omega_{1}\right)}+\frac{1}{\left(\Omega_{n 1}+\omega_{2}\right)\left(\Omega_{n 2}-\omega_{2}\right)}\right)\right]\right\} .
\end{aligned}
$$

Because we are considering the situation $\omega_{1}+\omega_{2}$ $\simeq \Omega_{12}$, only the term involving $e^{-i\left(\Phi_{1}+\Phi_{2}\right)}$ in Eq. (B6) need be retained. We will now display only the essential results. For the vector model,

$$
\begin{aligned}
& \gamma_{1}=-\kappa \mathcal{E}_{1} \mathcal{E}_{2}, \\
& \gamma_{2}=0, \\
& \gamma_{3}=\Omega_{12}-\left(\omega_{1}^{\prime}+\omega_{2}^{\prime}\right)+\left(\Delta E_{1}-\Delta E_{2}\right) / \hbar,
\end{aligned}
$$

with

$$
\begin{aligned}
& \omega_{1}^{\prime}=\omega_{1}+\partial \phi_{1} / \partial t, \\
& \omega_{2}^{\prime}=\omega_{2}+\partial \phi_{2} / \partial t,
\end{aligned}
$$

and

$$
\kappa=\frac{1}{\hbar^{2}}\left|\sum_{n} p_{1 n} p_{n 2}\left(\frac{1}{\Omega_{n 2}-\omega_{1}}+\frac{1}{\Omega_{n 2}-\omega_{2}}\right)\right| .
$$

The polarization $\vec{p}(t)$ for a single atom has the form

$$
\overrightarrow{\mathrm{p}}(t)=\overrightarrow{\mathrm{p}}_{\mathrm{I}}(t)+\overrightarrow{\mathrm{p}}_{\mathrm{II}}(t)+\overrightarrow{\mathrm{p}}_{\mathrm{III}}(t) \text {. }
$$

The individual components of the polarization are equal to

$$
\begin{aligned}
\overrightarrow{\mathrm{p}}_{\mathrm{I}}(t)= & \frac{1}{2}\left[\chi_{1}\left(\omega_{1}\right)\left(1+r_{3}\right)+\chi_{2}\left(\omega_{1}\right)\left(1-r_{3}\right)\right] \overrightarrow{\mathrm{E}}_{1} \\
& +\frac{1}{2}\left[\chi_{1}\left(\omega_{2}\right)\left(1+r_{3}\right)+\chi_{2}\left(\omega_{2}\right)\left(1-r_{3}\right)\right] \overrightarrow{\mathrm{E}}_{2}, \quad(\mathrm{~B} 10 \mathrm{a}) \\
\overrightarrow{\mathrm{p}}_{\mathrm{II}}(t)= & \operatorname{Re} \frac{1}{2} \hbar \kappa\left(r_{1}-i r_{2}\right)(\hat{x}+i \hat{y})\left(\mathcal{E}_{1} e^{-i \Phi_{2}}+\mathcal{E}_{2} e^{\left.-i \Phi_{1}\right)},\right.
\end{aligned}
$$

and

$$
\overrightarrow{\mathrm{p}}_{\mathrm{III}}(t)=0 \text {. }
$$

Using the AF solutions for the components of $\vec{r}$, we can rewrite Eqs. (B10) $\mathrm{as}^{38}$

$$
\begin{aligned}
\overrightarrow{\mathrm{p}}_{\mathrm{I}}(t)= & \frac{1}{2}\left[\chi_{1}\left(\omega_{1}\right)(1 \mp \cos \theta)+\chi_{2}\left(\omega_{1}\right)(1 \pm \cos \theta)\right] \overrightarrow{\mathrm{E}}_{1} \\
& +\frac{1}{2}\left[\chi_{1}\left(\omega_{2}\right)(1 \mp \cos \theta)+\chi_{2}\left(\omega_{2}\right)(1 \pm \cos \theta)\right] \overrightarrow{\mathrm{E}}_{2},
\end{aligned}
$$

and

$$
\begin{aligned}
\overrightarrow{\mathrm{p}}_{\mathrm{II}}(t) & =\frac{1}{2} \operatorname{Re} \chi^{(3)}(\hat{x}+i \hat{y})\left(\mathcal{E}_{1} e^{-i \Phi_{2}}+\mathcal{E}_{2} e^{-i \Phi_{1}}\right) \\
& \times\left(1+\frac{i}{\gamma_{3}} \frac{\partial}{\partial t}\right) \mathcal{E}_{1} \mathcal{E}_{2}\left|\frac{\delta}{\gamma}\right|
\end{aligned}
$$


where

$$
\begin{gathered}
\chi^{(3)=\frac{1}{\hbar^{3}} \sum_{m, n}} \frac{p_{2 n} p_{n 1} p_{1 m} p_{m 2}}{\delta}\left(\frac{1}{\Omega_{m 2}-\omega_{1}}+\frac{1}{\Omega_{m 2}-\omega_{2}}\right) \\
\times\left(\frac{1}{\Omega_{n 2}-\omega_{1}}+\frac{1}{\Omega_{n 2}-\omega_{2}}\right)
\end{gathered}
$$

In the limit where $\pi-\theta \ll 1$ and $\mathcal{E}_{1}, \mathcal{E}_{2}, \Phi_{1}$, and $\Phi_{2}$ are constant, Eqs. (B11) can be reduced to the simple form

$$
\begin{aligned}
& \overrightarrow{\mathrm{p}}_{\mathrm{I}}(t)=\chi_{2}\left(\omega_{1}\right) \overrightarrow{\mathrm{E}}_{1}+\chi_{2}\left(\omega_{2}\right) \overrightarrow{\mathrm{E}}_{2}, \\
& \overrightarrow{\mathrm{p}}_{\mathrm{II}}(t)=\frac{1}{2} \chi^{(3)}\left(\mathcal{E}_{2}^{2} \overrightarrow{\mathrm{E}}_{1}+\mathcal{E}_{1}^{2} \overrightarrow{\mathrm{E}}_{2}\right) .
\end{aligned}
$$

\section{Single-frequency case}

Here we treat the case where the second harmonic of the input field is near resonant to $\Omega_{12}$. Let the input field be

$$
\overrightarrow{\mathrm{E}}=\mathcal{E}(\hat{x} \cos \Phi+\hat{y} \sin \Phi) \text {, }
$$

with

$$
\Phi=\omega t+\phi-k z
$$

and

$$
2 \omega \simeq \Omega_{12} .
$$

Most of the expressions in Appendix B1 apply if we make the following changes: $\mathcal{E}_{1} \rightarrow \mathcal{E}, \mathcal{E}_{2} \rightarrow 0$, $\omega_{2} \rightarrow \omega$, and $\omega_{1} \rightarrow \omega$. However, for completeness we will display the pertinent equations. For the vector model,

$$
\begin{aligned}
& \gamma_{1}=-\kappa \mathcal{E}^{2}, \\
& \gamma_{2}=0, \\
& \gamma_{3}=\Omega_{12}-2 \omega^{\prime}+\left(\Delta E_{1}-\Delta E_{2}\right) / \hbar
\end{aligned}
$$

with

$$
\omega^{\prime}=\omega+\partial \phi / \partial t
$$

and

$$
\kappa=\frac{1}{\hbar^{2}}\left|\sum_{n} \frac{p_{1 n} p_{n 2}}{\Omega_{n 2}-\omega}\right|
$$

and the optical Stark shifts for states $|1\rangle^{\prime}$ and $|2\rangle^{\prime}$ are given by Eqs. (B4) with $\mathcal{E}_{2}=0$.

The expressions for the polarization are

$$
\begin{aligned}
& \overrightarrow{\mathrm{p}}_{\mathrm{I}}(t)=\frac{1}{2}\left[\chi_{1}\left(1+r_{3}\right)+\chi_{2}\left(1-r_{3}\right)\right] \overrightarrow{\mathrm{E}}, \\
& \overrightarrow{\mathrm{p}}_{\mathrm{II}}(t)=\operatorname{Re} \hbar \kappa\left(r_{1}-i r_{2}\right)(\hat{x}+i \hat{y}) \mathcal{E} e^{-i \Phi}, \\
& \overrightarrow{\mathrm{p}}_{\mathrm{III}}(t)=0 .
\end{aligned}
$$

Using the AF solutions for the components of $\vec{r}$, we can rewrite Eqs. (B17) as ${ }^{38}$

$$
\overrightarrow{\mathrm{p}}_{\mathrm{I}}(t)=\frac{1}{2}\left[\chi_{1}(1 \mp \cos \theta)+\chi_{2}(1 \pm \cos \theta)\right] \overrightarrow{\mathrm{E}},
$$

$$
\overrightarrow{\mathrm{p}}_{\mathrm{II}}(t)=\frac{1}{2} \operatorname{Re} \chi^{(3)}(\omega)(\hat{x}+i \hat{y}) \mathcal{E} e^{-i \Phi}\left(1+\frac{i}{\gamma_{3}} \frac{\partial}{\partial t}\right) \mathcal{E}^{2}\left|\frac{\delta}{\gamma}\right|,
$$

where

$$
\chi^{(3)}(\omega)=\frac{2}{\hbar^{3}} \sum_{m, n} \frac{p_{2 n} p_{n 1} p_{1 m} p_{m 2}}{\delta\left(\Omega_{m 2}-\omega\right)\left(\Omega_{n 2}-\omega\right)} .
$$

In the limit where $\pi-\theta<<1$ and $\mathcal{E}$ and $\Phi$ are constant Eqs. (B18) can be reduced to the simple form

$$
\overrightarrow{\mathrm{p}}_{\mathrm{I}}(t)=\chi_{2} \overrightarrow{\mathrm{E}}
$$

and

$$
\overrightarrow{\mathrm{p}}_{\mathrm{II}}(t)=\frac{1}{2} \chi^{(3)}(\omega) \mathcal{E}^{2} \overrightarrow{\mathrm{E}}
$$

\section{APPENDIX C: TWO-PHOTON RESONANTLY ENHANCED FREQUENCY UP-CONVERSION}

Two-photon resonantly enhanced nonlinear mixing in atomic vapors has recently been demonstrated. ${ }^{10,12}$ The method of Hodgson, Sorokin, and Wynne ${ }^{10}$ utilizes one laser source tuned to be resonant with a two-photon transition, i.e., $2 \omega_{1}$ $\simeq \Omega_{12}$. A second source at the frequency $\omega_{2}$ then interacts with the atoms to produce output at $2 \omega_{1}$ $+\omega_{2}$. It is convenient to use circularly polarized light in these mixing schemes, since third-harmonic generation at $3 \omega_{1}$ or $3 \omega_{2}$ is then forbidden. The desired mixing frequency, $2 \omega_{1}+\omega_{2}$, will still be generated provided the sense of circular polarization of the two beams at $\omega_{1}$ and $\omega_{2}$ are opposite to each other. We therefore choose the input field to be

$$
\overrightarrow{\mathrm{E}}=\overrightarrow{\mathrm{E}}_{1}+\overrightarrow{\mathrm{E}}_{2}
$$

with

$$
\overrightarrow{\mathrm{E}}_{1}=\mathcal{E}_{1}\left(\hat{x} \cos \Phi_{1}+\hat{y} \sin \Phi_{1}\right)
$$

and

$$
\overrightarrow{\mathrm{E}}_{2}=\mathcal{E}_{2}\left(\hat{x} \cos \Phi_{2}-\hat{y} \sin \Phi_{2}\right) .
$$

The electric dipole matrix elements are taken to be the same as in Appendix B.

Because $2 \omega_{1} \simeq \Omega_{12}$, the response of the two-photon transition is primarily determined by only the radiation at $\omega_{1}$. Hence, the appropriate vector model for these processes is the single-frequency model for circularly polarized light given in Appendix B2, except for the Stark shifts $\Delta E_{1}$ and $\Delta E_{2}$, which are given by Eqs. (B4) with $\omega_{2}$ replaced by $-\omega_{2}$. The expressions for the $\gamma$ vector are given in Eqs. (B15). The up-converted frequency appears when we make the transformations in Eq. (50) from the double-primed representation to the laboratory frame with the appropriate transformation operators $U$ and $R$ derived for circularly polarized light. 
In terms of the vector model components $r_{1}$ and $r_{2}$, the polarization $\overrightarrow{\mathrm{p}}_{\mathrm{III}}(t)$ for a single atom is found to be

$$
\begin{aligned}
\overrightarrow{\mathrm{p}}_{\mathrm{III}}(t)=\operatorname{Re} \sum_{n} & \frac{p_{2 n} p_{n 1}}{2 \hbar} \mathcal{E}_{2} e^{-i\left(2 \Phi_{1}+\Phi_{2}+\beta\right)} \\
& \times\left(\frac{1}{\Omega_{n 2}+\omega_{2}}+\frac{1}{\Omega_{n 2}-\left(2 \omega_{1}+\omega_{2}\right)}\right) \\
& \times(\hat{x}+i \hat{y})\left(r_{1}-i r_{2}\right) .
\end{aligned}
$$

Explicit expressions can be obtained for this polarization by solving the equations of motion for $r_{1}$ and $r_{2}$. Using the AF solutions for the components of $\vec{r}$ given by Eqs. (58)-(61), we obtain

$$
\begin{aligned}
\overrightarrow{\mathrm{p}}_{\mathrm{III}}(t)= & \frac{1}{2} \operatorname{Re} \chi^{(3)}\left(2 \omega_{1}+\omega_{2}\right)(\hat{x}+i \hat{y}) \mathcal{E}_{2} e^{-i\left(2 \Phi_{1}+\Phi_{2}\right)} \\
& \times\left(1+\frac{i}{\gamma_{3}} \frac{\partial}{\partial t}\right) \mathcal{E}_{1}^{2}\left|\frac{\delta}{\gamma}\right|,
\end{aligned}
$$

where

$$
\begin{aligned}
& \chi^{(3)}\left(2 \omega_{1}+\omega_{2}\right) \\
& \quad=\frac{1}{\hbar^{3}} \sum_{m, n} \frac{p_{2 n} p_{n 1} p_{1 m} p_{m 2}}{\delta\left(\Omega_{m 2}-\omega_{1}\right)}\left(\frac{1}{\Omega_{n 2}+\omega_{2}}+\frac{1}{\Omega_{n 2}-\left(2 \omega_{1}+\omega_{2}\right)}\right)
\end{aligned}
$$

and

$$
\delta=\Omega_{12}-2 \omega_{1}
$$

Again in the limit where $\pi-\theta<<1$ and $\mathcal{E}_{1}, \mathcal{E}_{2}$, $\Phi_{1}$, and $\Phi_{2}$ are constant, Eq. (C3) can be reduced to the simple form of Ref. 12,

$$
\overrightarrow{\mathrm{p}}_{\mathrm{III}}=\frac{1}{2} \operatorname{Re} \chi^{(3)}\left(2 \omega_{1}+\omega_{2}\right)(\hat{x}+i \hat{y}) \mathcal{E}_{1}^{2} \mathcal{E}_{2} e^{-i\left(2 \Phi_{1}+\Phi_{2}\right)} .
$$

*Work of this author was partially supported by the U. S. Office of Naval Research.

${ }^{1}$ R. P. Feynman, F. L. Vernon, Jr., and R. W. Hellwarth, J. Appl. Phys. 28, 49 (1957).

${ }^{2}$ M. Takatsuji, Phys. Rev. A $\underline{4}, 808$ (1971).

${ }^{3}$ R. G. Brewer and E. L. Hahn, Phys. Rev. A 11, 1641 (1975).

${ }^{4}$ D. Grischkowsky, Phys. Rev. Lett. 24, 866 (1970).

${ }^{5}$ D. Grischkowsky and J. A. Armstrong, Phys. Rev. A $\underline{6}$, 1566 (1972).

${ }^{6}$ D. Grischkowsky, Phys. Rev. A 7, 2096 (1973).

${ }^{7}$ D. Grischkowsky, Eric Courtens, and J. A. Armstrong, Phys. Rev. Lett. 31, 422 (1973).

${ }^{8} \mathrm{D}$. Grischkowsky, in Laser Applications to Optics and Spectroscopy, Vol. II of Physics of Quantum Electronics Series, edited by S. F. Jacobs, M. Sargent, III, J. F. Scott, and M. O. Scully (Addison-Wesley, Reading, Mass., 1975).

${ }^{9}$ J. A. Armstrong, N. Bloembergen, J. Ducuing, and P. S. Pershan, Phys. Rev.127, 1918 (1962).

${ }^{10}$ R. T. Hodgson, P. P. Sorokin, and J. J. Wynne, Phys. Rev. Lett. 32, 343 (1974).

${ }^{11}$ C. S. Chang, Phys. Rev. A $\underline{9}, 1769$ (1974).

${ }^{12}$ K. M. Leung and J. F. Ward, and B. J. Orr, Phys. Rev. A 9,2440 (1974).

${ }^{13}$ S. R. Hartmann, IEEE J. Quantum Elect. QE-4, 802 (1968).

${ }^{14}$ E. M. Belenov and I. A. Poluektov, Zh. Eksp. Teor. Fiz. 56, 1407 (1969) [Sov. Phys.- JETP 29, 754 (1969)]. ${ }^{15}$ R. L. Shoemaker and R. G. Brewer, Phys. Rev. Lett. 28, 1430 (1972).

${ }^{16}$ B. J. Orr and J. F. Ward, Mol. Phys. 20, 513 (1971).

${ }^{17}$ R. B. Miles and S. E. Harris, IEEE J. Quantum Electronics QE-9, 470 (1973).

${ }^{18}$ Naohiro Tan-no, Ken-ichi Yokoto, and Humio Inaba, Phys. Rev. Lett. 29, 1211 (1972).

${ }^{19}$ I. A. Poluektov, $\overrightarrow{\mathrm{Yu}} . \mathrm{M}$. Popov, and V. S. Roitberg, Zh. Eksp. Teor. Fiz. Pis'ma Red. 18, 638 (1973) [JETP Lett. 18,373 (1973)].

${ }^{20}$ I. A. Poluektov, Yu. M. Popov, and V. S. Roitberg, Zh.
Eksp. Teor. Fiz. Pis'ma Red. 20, 533 (1974) [JETP Lett. 20, 243 (1974)].

${ }^{21}$ M. Takatsuji, Phys. Rev. A 11, 619 (1975).

${ }^{22}$ Naohiro Tan-no, Ken-ichi Yokoto, and Humio Inaba, J. Phys. B $\underline{8}, 339$ (1975); $\underline{8}, 349$ (1975).

${ }^{23}$ Hiroki Nakatsuka, Jumpei Okada, and Masahiro Matsuoka, J. Phys. Soc. Jpn. 37, 1406 (1974).

${ }^{24} \mathrm{R}$. Weingarten, Ph.D. thesis (Columbia University, 1972) (unpublished).

${ }^{25} \mathrm{~W}$. Heitler, The Quantum Theory of Radiation (Oxford U. P., London, 1954), 3rd ed., p. 146.

${ }^{26}$ A. M. Bonch-Bruevich and V. A. Khodovoi, Usp. Fiz. Nauk 93, 71 (1967) [Sov. Phys.-Usp. 10, 637 (1968)].

${ }^{27}$ P. F. Liao and J. E. Bjorkholm, Phys. Rev. Lett. $\underline{34}$, 1 (1975).

${ }^{28} \mathrm{In}$ terms of the notation of Ref. $36,\left|1_{m}\right\rangle=|1\rangle^{\prime}$ and $\left|2_{m}\right\rangle=|2\rangle^{\prime}$. Also, $a_{n 1}=U_{n i}^{-1}$, and $a_{n 2}=U_{n 2}^{-1}$.

${ }^{29}$ David Bohm, Quantum Theory (Prentice-Hall, Englewood Cliffs, N.J., 1951), p. 448.

${ }^{30}$ I. D. Abella, N. A. Kurnit, and S. R. Hartmann, Phys. Rev. Lett. 13, 567 (1964); Phys. Rev. 141, 391 (1966).

${ }^{31}$ G. B. Hocker and C. L. Tang, Phys. Rev. Lett. 21, 591 (1968); Phys. Rev. 184, 356 (1969).

${ }^{32}$ E. B. Treacy, Phys. Lett. 27A, 421 (1968).

${ }^{33}$ E. B. Treacy and A. J. DeMaria, Phys. Lett. 29A, 369 (1969).

${ }^{34}$ M. M. T. Loy, Phys. Rev. Lett. 32, 814 (1974).

${ }^{35}$ S. L. McCall and E. L. Hahn, Phys. Rev. Lett. 18, 908 (1967); Phys. Rev. 183, 457 (1969); Phys. Rev. A 2,861 (1970).

${ }^{36}$ D. Grischkowsky and M. M. T. Loy, Phys. Rev. A 12 , 1117 (1975).

${ }^{37}$ M. D. Crisp, Phys. Rev. A $\underline{8}, 2128$ (1973).

${ }^{38}$ If in the Bloch equations (43) we had introduced the transverse relaxation time $T_{2}, \gamma_{2}$ would be given by [see Ref. 8, Eq. (30)] $r_{2}=-\left(1 / \gamma_{3}\right)\left(1 / T_{2}+\partial / \partial t\right) r_{1}$, instead of our Eq. (61). For this case the operator 1 $+\left(i / \gamma_{3}\right)(\partial / \partial t)$ is replaced by $1+\left(i / \gamma_{3}\right)\left(1 / T_{2}+\partial / \partial t\right)$ in all the polarization formulas.

${ }^{39}$ E. A. Stappaerts, Phys. Rev. A 11, 1664 (1975). 
${ }^{40}$ We note the recent self-defocusing experiment, where the nonlinearity was of this form and was due to the two-photon resonance: R. H. Lehmberg, J. Reintjes, and R. C. Eckardt, Appl. Phys. Lett. 25, 374 (1974). ${ }^{41}$ This is the same system as used in the recent experimental study of coherence in two-photon excited $\mathrm{Rb}$ vapor by Nakatsuka et al. (Ref. 23).

${ }^{42}$ O. S. Heavens, J. Opt. Soc. Am. 51, 1058 (1961).

${ }^{43}$ D. Grischkowsky, Appl. Phys. Lett. 25, 566 (1974).

${ }^{44}$ Because the example makes use of resonant enhancement, a more complete calculation is required to properly treat the single-photon resonance from the twophoton ground state $|2\rangle$ to the intermediate state $|n\rangle$. The main reshaping effect neglected by Eqs. (81) is the self-phase modulation caused by the nonlinear dielectric constant $\epsilon_{2}^{(1)}$ of the single-photon transition (Ref. 8). This type of problem is similar to that of including the nonlinearity of the host material in the reshaping problem. Including this effects adds the quantity $-\frac{1}{2}(\omega / c) \epsilon_{2}^{(1)}(\partial / \partial t) \mathcal{E}^{2}$ to the right-hand side of Eq. (81b), where (Ref. 8) $\epsilon_{2}^{(1)}=-4 \pi N p_{2 n}^{4} / \hbar^{3}\left(\Omega_{n 2}-\omega\right)^{3}$. Equation (81a) remains the same. Recalculating the pulse reshaping for this situation gives results similar to Fig. 5, except the required propagation distance is now $40 \mathrm{~cm}$.

${ }^{45}$ L. Casperson and A. Yariv, Phys. Rev. Lett. 26, 293 (1971). 